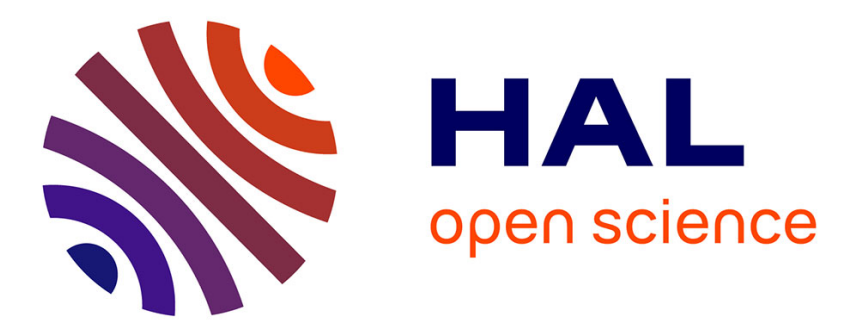

\title{
Identifiability of single crystal plasticity parameters from residual topographies in Berkovich nanoindentation on FCC nickel
}

\author{
Emile Renner, Alexandre Bourceret, Yves Gaillard, Fabien Amiot, Patrick \\ Delobelle, Fabrice Richard
}

\section{To cite this version:}

Emile Renner, Alexandre Bourceret, Yves Gaillard, Fabien Amiot, Patrick Delobelle, et al.. Identifiability of single crystal plasticity parameters from residual topographies in Berkovich nanoindentation on FCC nickel. Journal of the Mechanics and Physics of Solids, 2020, 138, pp.103916 (20). 10.1016/j.jmps.2020.103916 . hal-02980605

\section{HAL Id: hal-02980605 https://hal.science/hal-02980605}

Submitted on 27 Oct 2020

HAL is a multi-disciplinary open access archive for the deposit and dissemination of scientific research documents, whether they are published or not. The documents may come from teaching and research institutions in France or abroad, or from public or private research centers.
L'archive ouverte pluridisciplinaire HAL, est destinée au dépôt et à la diffusion de documents scientifiques de niveau recherche, publiés ou non, émanant des établissements d'enseignement et de recherche français ou étrangers, des laboratoires publics ou privés. 


\title{
Identifiability of single crystal plasticity parameters from residual topographies in Berkovich nanoindentation on FCC nickel
}

\author{
E. Renner ${ }^{1}$, A. Bourceret ${ }^{2}$, Y. Gaillard ${ }^{2}$, F. Amiot ${ }^{2}$, P. Delobelle ${ }^{2}$ and F. Richard ${ }^{2}$ \\ ${ }_{1}$ CEA Le Ripault, DMAT/SRCC/LMC, Monts, France
}

2Univ. Bourgogne Franche-Comté, Institut FEMTO-ST, CNRS/UFC/ENSMM/UTBM, Département Mécanique Appliquée, Besançon, France

Keywords: Crystal plasticity (A), Berkovich nanoindentation (B), Parameter identifiability (C) Corresponding author, e-mail address: fabrice.richard@univ-fcomte.fr

\begin{abstract}
The information richness of imprints topographies obtained after Berkovich nanoindentation tests at grain scale is assessed for identifying all or part of the parameters of a single crystal plasticity law. In a previous paper (Renner et al., 2016), the strong potential of imprints topographies has been shown through a large experimental campaign conducted on nickel samples. A 3D crystal plasticity finite element modelling (CPFEM) of the nanoindentation experiment using the Méric-Cailletaud has also showed a large sensitivity of residual topographies to the indenter/grain orientation and to the plastic parameters, including the interaction matrix coefficients specifying the interactions between dislocations on different slip systems. This makes imprints topographies very good candidates to provide information for the single crystal parameters identification. The present paper focuses on the Méric-Cailletaud law parameters identifiability using residual topographies. A method is built to define the best well-posed inverse problem to ensure the parameters identification using a crystal plasticity finite element modelling updating (CPFEMU) method. An identifiability index proposed by Richard et al. (Richard et al., 2013) for measuring the information richness of the indentation curve is extended to the analysis of residual topographies. This index quantifies the possibility to achieve a stable/unstable solution using an inverse method. For the studied behaviour, the results show that eight of the nine Méric-Cailletaud law parameters can be identified using three topographies.
\end{abstract}

\section{Introduction}

Material parameters identification for single crystal plasticity laws remains a topical issue for a better understanding of metal behaviour at grain or polycrystalline scale (Méric et al., 1994; Fivel et al., 1997; Gérard, 2008; Gérard et al., 2013, 2009; Guilhem, 2011; Schwartz, 2011; 
Zambaldi et al., 2012; Guery, 2014; Guery et al., 2014; Tasan et al., 2014b, 2014a; Zambaldi et al., 2015). At the dislocation scale, some authors approached this issue using dislocation dynamics simulations (Fivel, 1997; Fivel et al., 1998; Forest and Fivel, 2001; Madec, 2001; Devincre et al., 2006). At the grain scale, the laws are also very complete and thus challenging when it comes to the material parameters identification from experimental data using inverse method. In this context, the often-missing concept of ill-posed problem, or identifiability, is essential to assess the stability and the uniqueness of the solution. The Méric-Cailletaud law is easily and effectively implemented in FE codes like ZeBuLon (Burlet and Cailletaud, 1991) [http://www.zset-software.com]. Strong nonlinearities and couplings between the dissipative phenomena during indentation make the identification of the parameters of the MéricCailletaud single crystal plasticity law very difficult, especially for the six interaction matrix components. These six parameters define the dislocation interactions hardening in the 12 FCC slip systems. Most recently, some works used FEM updating for identifying these interaction components, but one can notice some limitations. Méric et al performed tensile tests on single and bi-crystals, which may be expensive and limited to materials that can be obtained macroscopically (Méric et al., 1994). Gérard et al. performed tensile tests on preloaded polycrystalline samples. These complex loadings activate the slip systems interactions. Even if most of isotropic hardening law parameters are identified, it does not provide enough information and some interaction components remain inaccessible (Gérard, 2008; Gérard et al., 2009). Guery et al. performed in-situ tensile tests on polycrystalline samples in which they measure macroscopic loads and displacement fields by digital image correlation (Guery, 2014; Guery et al., 2014). The authors have the merit of assessing the interaction components identifiability before identifying them. Besides the fact that the techniques used are quite complex, a gap subsists between experimental and numerical results. Moreover, the two latest studies are performed at the polycrystalline scale and must take the scale transition rules into account.

Based on the principle of hardness test, the instrumented indentation test, or nanoindentation test, offers three major advantages for the identification of single crystal plasticity laws. Firstly, it is easy of use. It performs continuous measurement of the applied load $P$ on the indenter and of the indentation depth $h$. Thus, the indentation curve $(P-h)$ can be used to locally extract an elastic modulus and hardness when interpreted in the framework proposed in Sneddon et al. (Harding and Sneddon, 1945; Sneddon, 1965), Oliver and Pharr (Oliver and Pharr, 1992) and Vlassak and Nix works (Vlassak and Nix, 1993, 1994). Secondly, the stress field generated by the Berkovich indenter (three-sided based pyramid) penetration is multiaxial. One can think that the nanoindentation test activates simultaneously a large number of slip systems. Finally, it provides a direct mechanical measurement at intra-granular scale 
without any neighbouring grains interaction if the average grain size is large enough compared to the imprint size.

Indentation curves have been used by some authors for the identification of elastic-plastic parameters at a macroscopic scale. They mostly concluded that it is usually impossible to correctly identify plastic parameters for any kind of indenter shape (conical, pyramidal, spherical), even using "simple" elastic-plastic laws involving only two plastic parameters as the yield stress $\sigma_{y}$ and the work-hardening exponent $n$ of a power hardening law. Solutions are often unstable. Cheng and Cheng (Cheng and Cheng, 1999) first pointed out this issue. Some authors have then numerically proved that, assuming that the Young modulus $E$ of an isotropic material is known, both plastic parameters $\left(\sigma_{y}, n\right)$ are not identifiable using the entire conical indentation curve (Capehart and Cheng, 2003; Tho et al., 2004; Alkorta et al., 2005). They showed that different materials governed by this law can lead to indistinguishable indentation curves (Figure 1). To yield a unique solution, the dual nanoindentation technique using different conical indenter tips with half angles ranging from $60^{\circ}$ to $80^{\circ}$ has been proposed (Chen et al., 2007). But once again, they showed the existence of "mystical" materials which give almost indistinguishable indentation curves. This "mystical" material identification issue has been solved by some authors. Zhao et al. performed indentations on thin films which were indistinguishable by bulk indentation (Zhao et al., 2007). Thanks to the substrate effect, the elastoplastic properties can then be derived from an inverse analysis. Another solution was proposed by $\mathrm{Ma}$ et al. which is close to the approach considered in the present paper (Ma et al., 2012). They succeeded to correctly identify "mystical" materials using the indentation curves but also the residual imprint topographies in the inverse analysis. Other authors have examined this difficulty and have shown that non-uniqueness is an extreme case of instability of the solution (Cao, 2004; Phadikar et al., 2013).
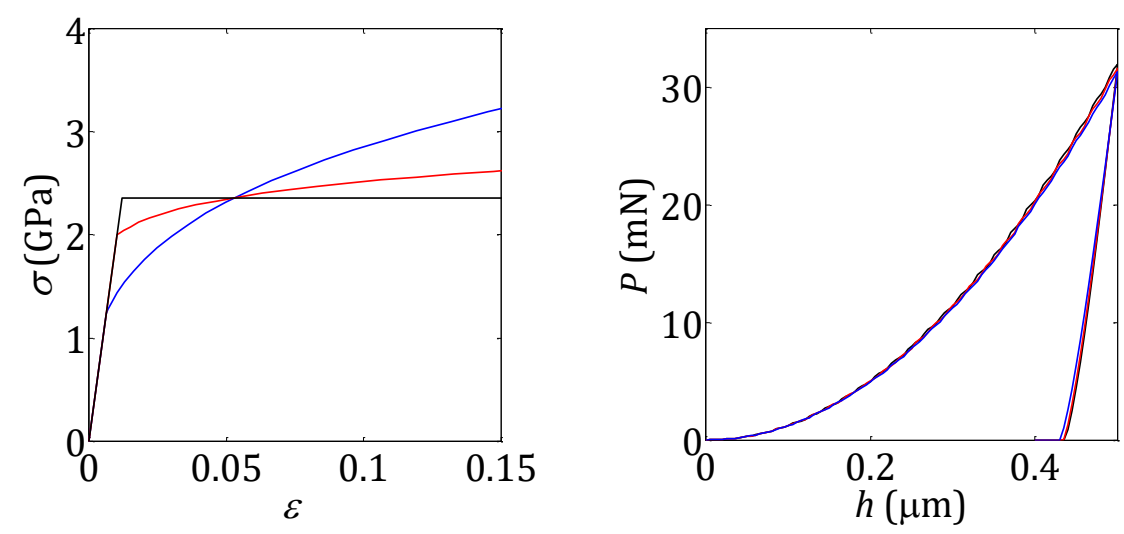

Figure 1 - Indistinguishable conical indentation curves $\left(68^{\circ}\right)$ simulated using three macroscopic elastic-plastic material behaviours.

"Highly plastic" behaviour (small ratio $\sigma_{y} / E$ ) (Cheng and Cheng, 1999). 
Thus, the parameter identification of the Méric-Cailletaud law using the indentation curve is not a possible scenario since the identification of much simpler elastic-plastic laws at macroscopic scale is an issue. Bolzon et al (Bolzon et al., 2004) and Bocciarelli et al (Bocciarelli et al., 2005) used both the indentation curve and the imprint mapping for FEMU process. Zambaldi and Raabe said that "For the indentation of crystals, the topography of the free surface around the indent, the pile-up profile, can be used as a fingerprint of the underlying crystal deformation processes." (Zambaldi and Raabe, 2010). Indeed, pile-ups around the indentation imprint contain precious information for parameters identification. Targeting an extension of these results to crystal plasticity laws, this contribution is intended to quantify the identifiability of all or part of the Méric-Cailletaud parameters at grain scale using topographies of imprints (Figure 2).
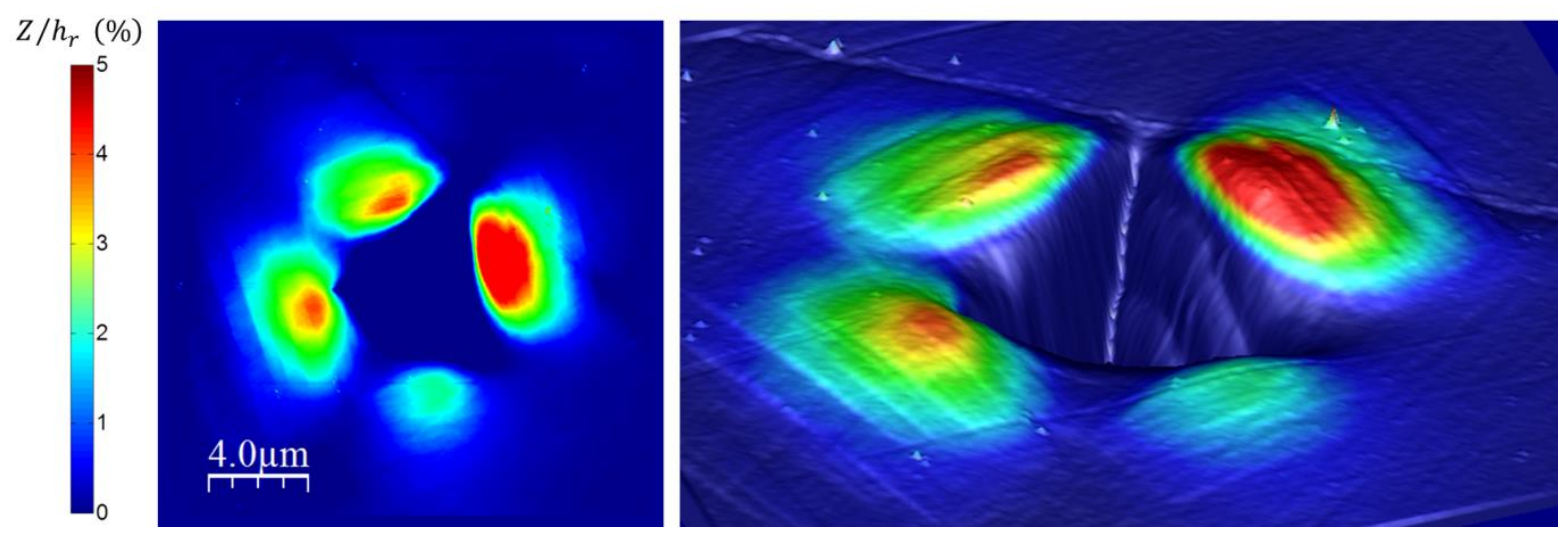

Figure 2 - Experimental AFM topography of a Berkovich indentation imprint on a polycrystalline annealed nickel sample, performed in a grain close to the [001] direction. Highlight of pile-ups at grain scale (Renner, 2016).

The paper is organized as follows. Firstly, the single crystal plasticity behaviour and the CPFEM of the nanoindentation test are described. Secondly, the identifiability method developed for measuring the information richness contained in indentation curves and residual topographies are described. Finally, the sensibility of the CPFEM and the identifiability of the plastic parameters are analysed and interpreted using one indentation curve only, one topography only and combinations of topographies.

\section{Single crystal plasticity framework}

Indentation experiments have been performed until $0.9 \mu \mathrm{m}$ depth, resulting in a residual imprint whose edge is $5.5 \mu \mathrm{m}$ long. They have been performed in large-grained samples. The average grain size is about $140 \mu \mathrm{m}$ which is large compared to the size of the indentations (Renner et al., 2016). Moreover, a particular attention has been paid to avoid indentations near the grain 
boundaries. Grain interaction effects are thus neglected and the use of the single crystal plasticity framework is justified.

\subsection{Constitutive laws}

An orthotropic stiffness tensor $\overline{\overline{\bar{C}}}$ is considered to model the nickel elastic response, described by three components $\left(C_{11}, C_{12}, C_{44}\right)$. The nanoindentation test imposes the use of a large deformation formulation due to the large strains reached in the contact region. A multiplicative decomposition of the transformation gradient $\overline{\overline{\boldsymbol{F}}}$ in an elastic $\overline{\overline{\boldsymbol{F}}}^{e}$ part and a plastic $\overline{\overline{\boldsymbol{F}}}^{p}$ part is adopted (Lee, 1969):

$$
\overline{\overline{\boldsymbol{F}}}=\overline{\overline{\boldsymbol{F}}}^{e} \cdot \overline{\overline{\boldsymbol{F}}}^{p}
$$

The plastic shear strain is assumed to occur only on each of the 12 FCC slip systems $\langle 110\rangle\{111\}$, characterized by the slip directions $\boldsymbol{l}^{s}$ and the normal to the slip planes $\boldsymbol{n}^{s}$ :

$$
\dot{\overline{\boldsymbol{F}}}^{p} \cdot \overline{\overline{\boldsymbol{F}}}^{p^{-1}}=\sum_{s=1}^{12} \dot{\gamma}^{s} \boldsymbol{l}^{s} \otimes \boldsymbol{n}^{s}
$$

The inelastic flow $\dot{\gamma}^{s}$ on system $s$ is chosen as a Norton power law to describe the material visco-plasticity, based on the Méric-Cailletaud size-independent single crystal plasticity law:

$$
\dot{\gamma}^{s}=\left\langle\frac{\left|\tau^{s}\right|-r^{s}-r_{0}}{K}\right\rangle^{n} \operatorname{sign}\left(\tau^{s}\right), \quad \text { with }\langle.\rangle=\max (0, .)
$$

where $K$ and $n$ are viscosity parameters and $r_{0}$ is the critical resolved shear stress. $r^{s}$ is the hardening on system $s$. The dislocations slipping initiation is governed by the resolved shear stress $\tau^{s}$ :

$$
\tau^{s}=\overline{\overline{\boldsymbol{\sigma}}}: \overline{\overline{\boldsymbol{m}}}^{s}, \quad \text { with } \overline{\overline{\boldsymbol{m}}}^{s}=\frac{1}{2}\left(\boldsymbol{l}^{s} \otimes \boldsymbol{n}^{s}+\boldsymbol{n}^{s} \otimes \boldsymbol{l}^{s}\right)
$$

where $\overline{\overline{\boldsymbol{\sigma}}}$ is the Cauchy stress tensor and $\overline{\overline{\boldsymbol{m}}}^{s}$ the Schmid tensor. The hardening $r^{s}$ has been chosen as purely isotropic:

$$
r^{s}=q \sum_{r=1}^{12} h^{s r}\left(1-\exp \left(-b v^{r}\right)\right), \quad \text { with } \dot{v}^{r}=\left|\dot{\gamma}^{r}\right|
$$

where $q$ and $b$ are material parameters and $v^{r}$ is the cumulated plastic slip on system $r . h^{s r}$ are the interaction matrix components which reflect the hardening associated to dislocation interactions between a primary system $s$ and a secondary one $r$. The interaction matrix is presented in Table 1. Due to the FCC structure symmetries, it is completely defined from 6 
independent parameters $\left(h_{1}, h_{2}, h_{3}, h_{4}, h_{5}, h_{6}\right)$ (Franciosi, 1985). Diagonal terms define selfhardening $\left(h_{1}\right)$ and off-diagonal terms define latent hardening: coplanar interaction $\left(h_{2}\right)$, Hirth locks $\left(h_{3}\right)$, colinear interaction $\left(h_{4}\right)$, glissile junctions $\left(h_{5}\right)$ and Lomer locks $\left(h_{6}\right)$.

\begin{tabular}{|c|c|c|c|c|c|c|c|c|c|c|c|c|}
\hline & 1 & 2 & 3 & 4 & 5 & 6 & 7 & 8 & 9 & 10 & 11 & 12 \\
\hline 1 & $h_{1}$ & $h_{2}$ & $h_{2}$ & $h_{4}$ & $h_{5}$ & $h_{5}$ & $h_{5}$ & $h_{6}$ & $h_{3}$ & $h_{5}$ & $h_{3}$ & $h_{6}$ \\
\hline 2 & & $h_{1}$ & $h_{2}$ & $h_{5}$ & $h_{3}$ & $h_{6}$ & $h_{4}$ & $h_{5}$ & $h_{5}$ & $h_{5}$ & $h_{6}$ & $h_{3}$ \\
\hline 3 & & & $h_{1}$ & $h_{5}$ & $h_{6}$ & $h_{3}$ & $h_{5}$ & $h_{3}$ & $h_{6}$ & $h_{4}$ & $h_{5}$ & $h_{5}$ \\
\hline 4 & & & & $h_{1}$ & $h_{2}$ & $h_{2}$ & $h_{6}$ & $h_{5}$ & $h_{3}$ & $h_{6}$ & $h_{3}$ & $h_{5}$ \\
\hline 5 & & & & & $h_{1}$ & $h_{2}$ & $h_{3}$ & $h_{5}$ & $h_{6}$ & $h_{5}$ & $h_{5}$ & $h_{4}$ \\
\hline 6 & & & & & & $h_{1}$ & $h_{5}$ & $h_{4}$ & $h_{5}$ & $h_{3}$ & $h_{6}$ & $h_{5}$ \\
\hline 7 & & & & & & & $h_{1}$ & $h_{2}$ & $h_{2}$ & $h_{6}$ & $h_{5}$ & $h_{3}$ \\
\hline 8 & & & & $\mathrm{~nm}$ & & & & $h_{1}$ & $h_{2}$ & $h_{3}$ & $h_{5}$ & $h_{6}$ \\
\hline 9 & & & & & & & & & $h_{1}$ & $h_{5}$ & $h_{4}$ & $h_{5}$ \\
\hline 10 & & & & & & & & & & $h_{1}$ & $h_{2}$ & $h_{2}$ \\
\hline 11 & & & & & & & & & & & $h_{1}$ & $h_{2}$ \\
\hline 12 & & & & & & & & & & & & $h_{1}$ \\
\hline
\end{tabular}

Table 1 - Interaction matrix of FCC structure.

\subsection{Virtual material}

The virtual material is a purely numerical material defined by 14 parameters $\left(C_{11}, C_{12}, C_{44}, K, n, r_{0}, q, b, h_{1}, h_{2}, h_{3}, h_{4}, h_{5}, h_{6}\right)$. The three components of the elastic tensor are chosen as $C_{11}=248 \mathrm{GPa}, C_{12}=153 \mathrm{GPa}, C_{44}=116 \mathrm{GPa}$ and the values of the viscosity parameters are set to $K=8 \mathrm{MPa} . \mathrm{s}^{1 / \mathrm{n}}$ and $n=7$ (Renner et al., 2016), which agrees with the commonly measured values for the Nickel (Rao and Varma, 1993). Using these values of $K$ and $n$, sensitivity of the indentation response to the viscosity parameters is low. Therefore, viscosity acts as a numerical regularization method of the rate-independent model. This is in agreement with the very low creep observed experimentally during the holding phase on the real annealed nickel sample in indentation test.

Finally, the plasticity model is a nine-parameter plastic model: $\boldsymbol{\theta}=\left(r_{0}, q, b, h_{1}, h_{2}, h_{3}, h_{4}, h_{5}, h_{6}\right)$. To analyse the identifiability of all or part of these nine parameters $\theta_{j}(j=1, \ldots, 9)$ from nanoindentation experiments, a virtual material $\boldsymbol{\theta}$ has been chosen (Table 2 ) and the identifiability results will be available in the close vicinity of this parameters set. The numerical process established to choose these parameters values is detailed in Renner et al. (Renner et al., 2016). The chosen virtual material promotes the $h_{3}$ interaction component (Hirth locks) and 
has been built so as to reproduce the strain hardening response of the real annealed nickel sample in tension. Note that the sum of the interaction matrix components over one line is $100=\left(h_{1}+2 h_{2}+2 h_{3}+h_{4}+4 h_{5}+2 h_{6}\right)$.

\begin{tabular}{l|ccc|cccccc} 
& \multicolumn{4}{|c|}{ hardening } & \multicolumn{5}{c}{ interaction } \\
\hline$j$ & 1 & 2 & 3 & 4 & 5 & 6 & 7 & 8 & 9 \\
\hline$\theta_{j}$ & $r_{0}$ & $q$ & $b$ & $h_{1}$ & $h_{2}$ & $h_{3}$ & $h_{4}$ & $h_{5}$ & $h_{6}$ \\
\hline value & $26.7 \mathrm{MPa}$ & $20.5 \mathrm{MPa}$ & 16 & 1 & 1 & 45 & 1 & 1 & 1
\end{tabular}

Table 2 - A virtual material (Renner et al., 2016). Nine plastic parameters $\theta_{j}$

\subsection{CPFEM of the nanoindentation experiment}

Identifiability analysis is based on a CPFEM of the nanoindentation experiment using the Méric-Cailletaud size-independent single crystal plasticity which is not detailed here, (refer to (Renner et al., 2016)). The CPFEM has been built using a generalized elastic-viscoplastic material model based on the infinitesimal strain theory (Z-set User commands Version 9.0, 2018). A corotational finite strain formulation based on an integrated rotation tensor has been used to take the material rotations into account (Ladeveze, 1980). The lattice is thus rotating with the material using the material spin tensor $\overline{\overline{\boldsymbol{W}}}=\left(\dot{\overline{\boldsymbol{F}}} \overline{\overline{\boldsymbol{F}}}^{-1}\right)^{\text {skew }}$ while the lattice rotation should be independent and occur in the elastic transformation part $\overline{\bar{F}}^{e}$ (Forest, 2010). The numerical integration of the material behaviour has been performed using the Runge-Kutta's second order method. The resolution method for the global matrix problem uses a full updated Newton-Raphson algorithm.

The contact problem is solved using a direct flexibility method (Francavilla and Zienkiewicz, 1975; Jean, 1995; Sachdeva and Ramakrishnan, 1981; Wronski, 1994). The contact between the bulk surface and the Berkovich indenter is modelled using the Coulomb law with a null friction coefficient. Indeed, the indenter geometry opening is quite large. The friction effect is thus negligible on the indentation curve (Taljat et al., 1998) and minor on the imprint topography compared to the hardening parameters.

The CPFEM of the nanoindentation experiment (direct problem) is parametrized using the vector $\boldsymbol{\Xi}=\left(\boldsymbol{\theta} ;[\mathrm{ij} \mathrm{k}], \alpha_{[i j k]}^{[a b c]}, h(t), t, X Y\right)$, where $\boldsymbol{\theta}$ is the vector of the nine plastic parameters (Table 2), [i j k] the Miller's indices (grain orientation), $\alpha_{[i j k]}^{[a b c]}$ the Berkovich tip azimuth, $h(t)$ the indentation depth, $t$ the time and the $X Y$ mapping region of the imprint topography. $\mathrm{A}$ coordinate system is related to the considered grain. The Miller's indices [i j k] define the first 
axis (perpendicular to the sample surface). The indices $[a b c]$ define the second axis which is arbitrary chosen in the sample surface plane. The third axis is deduced from the other to get an orthonormal coordinate system.

$\alpha_{[i j k]}^{[a b c]}$ is defined as the angle between the $[a b c]$-axis of the crystal coordinate system and the indenter base symmetry axis passing through the indenter corner contained in the coordinate system $1^{\text {st }}$ quadrant.

The Figure 3 illustrates a CPFEM simulation result: an indentation curve $\mathbf{P}$ and an imprint topography $\mathbf{Z}=\mathbf{Z}^{1}$. These results were obtained using the virtual material for $\mathbf{\Xi}=$ $\left(\boldsymbol{\theta} ;\left[\begin{array}{lll}1 & 0 & 1\end{array}\right], 74^{\circ}, t, h(t), t, X Y\right)$, virtual material $\theta_{j}$-values of (Table 2). Loading and unloading steps during the indentation tests last 30 seconds and holding at maximum force lasts 90 seconds. The mapping region $X Y$ is described by an area of $(20 \times 20) \mu \mathrm{m}^{2}$. A quantitative estimation of the plastic zone size can be given from the simulation. The cumulative plastic strain is found to vanish at a distance larger than six times the depth of the residual imprint. Indeed the size of the grains remains large compared to this value, validating the single crystal plasticity framework.

(a)

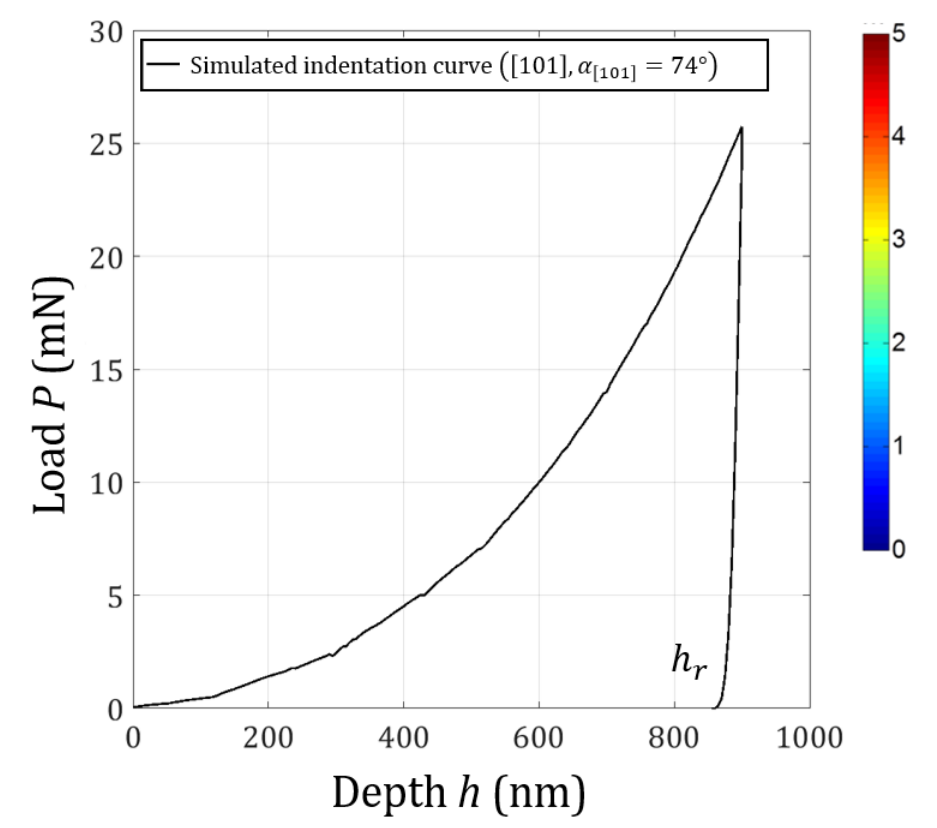

(b)

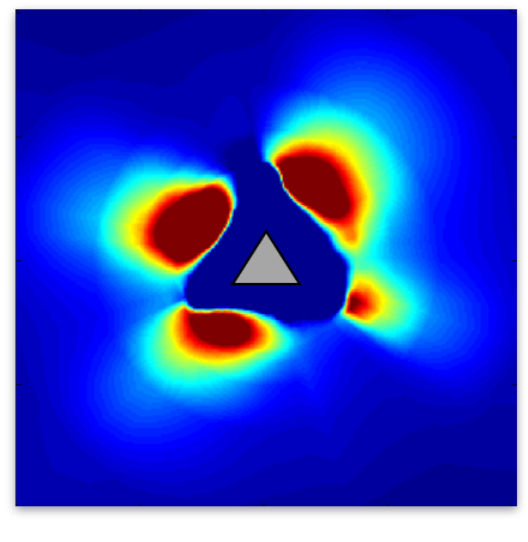

$[\overline{\mathbf{1 0 1}}]+\alpha_{[101]}^{[010]}=74^{\circ}$ [101]

Figure 3 - Nanoindentation CPFEM simulation performed in the [101] grain orientation with azimuth $\alpha_{[101]}^{[010]}=74^{\circ}$ using the virtual material: (a) indentation curve $\mathbf{P}$, (b) residual topography

$$
\mathbf{Z}=\mathbf{Z}^{1}
$$




\section{Plastic parameter identifiability method}

This section presents the numerical method used for estimating the identifiability of all or part of the 9 plastic parameters $\boldsymbol{\theta}=\left(r_{0}, q, b, h_{1}, h_{2}, h_{3}, h_{4}, h_{5}, h_{6}\right)$ from the indentation curves and topographies of imprints. These techniques is based on a CPFEM of the nanoindentation test (Renner et al., 2016) using the Méric-Cailletaud size-independent single crystal plasticity law. The identifiability index used for measuring the information richness in indentation curves and residual topographies is described. This index has been introduced for the first time by Richard et al. (Richard et al., 2013) for viscoelastic characterization from the sole indentation force, then in 2014 for plastic characterization from the sole indentation force (Pac et al., 2014). In this section, the identifiability index definition is extended to plastic characterization using residual topographies.

When some values of the plastic parameters $\theta_{j}(j=1, \ldots, 9)$ are unknown, they must be identified from experimental observations. The difficulty is then at first to well-pose the inverse problem. To build a well-posed inverse problem and identify some reliable values of all or part of the nine plastic parameters using a CPFEMU method, the information richness of numerical indentation curve $\mathbf{P}$, residual topography $\mathbf{Z}$ and combinations $\left(\mathbf{Z}^{1}, \ldots, \mathbf{Z}^{n_{\mathbf{z}}}\right)$ must be quantified. To quantify the reliability of plastic parameter values that could be obtained using a FEMU method, the completeness of data contained in $n_{\mathbf{z}}$ residual topographies $\left(\mathbf{Z}^{1}, \ldots, \mathbf{Z}^{n_{\mathbf{z}}}\right)$ considered separately or combined is quantified by extending the $I$-index formulation proposed by Richard et al. (Richard et al., 2013) for the analysis of the indentation curve. This index appears to be convenient to explore and investigate what are the optimal conditions to determine all or part of the nine plastic parameters of the material constitutive law from CPFEMU method. The analysis can be done before the updating process and therefore does not necessarily require the experimental measurements.

\subsection{Method using indentation curve}

To make the section easier to read, the formalism is first presented using the indentation curve $\mathbf{P}$ only for a given grain orientation [i j k], azimuth $\alpha_{[i j k]}^{[a b c]}$ and displacement-controlled $h(t)$ mode. The cost function is sampled in $T$ time steps $t_{k}$. The $I$-index proposed by Richard et al. (Richard et al., 2013) is a measure of the inverse problem conditioning and can be used with logarithmic notation (Pac et al., 2014):

$$
I_{\mathbf{p}}=\log _{10}\left(\frac{\lambda_{\max }}{\lambda_{\min }}\right)
$$


where $\lambda_{\max }$ and $\lambda_{\min }$ are respectively the maximum and the minimum eigenvalues of the dimensionless matrix $\overline{\mathbf{H}}^{\mathbf{P}}$ at the considered calculation point $\boldsymbol{\theta}$, respectively:

$$
\bar{H}_{i j}^{\mathbf{p}}=\sum_{k=1}^{T} \bar{S}_{k i}^{\mathbf{p}} \bar{S}_{k j}^{\mathbf{p}}
$$

$i$ and $j$ indicates the number of the parameters considered for the calculation (Table 2). $\bar{S}_{k i}^{\mathrm{P}}$ are the components of the force sensitivity vector to parameter $\theta_{j}$ :

$$
\bar{S}_{k j}^{\mathbf{p}}=\frac{\theta_{j}}{P_{\max } \sqrt{T}} \frac{\partial P_{k}}{\partial \theta_{j}}
$$

$P_{\max }$ (maximum simulated indentation force) and $\theta_{j}$ are used as scaling factors associated to the indentation force $P$ and the parameter $\theta_{j}$ itself, respectively. Each parameter $\theta_{j}$ is thus scaled by itself, which means that each parameter is sought using the same relative precision. Moreover, indented force is scaled by a constant $P_{\max }$, which means that an absolute uncertainty is considered on this variable.

For a chosen set of parameters, the matrix $\overline{\mathbf{H}}^{\mathbf{p}}$ contained the richness of the simulated indentation curve. By construction, this matrix contains information concerning the lack of sensitivity and the multicollinearity between the sensitivity vectors. These are two difficulties that can make the problem ill-posed (ill-conditioned). The lower the $I$-index, the better conditioned is the matrix, which means its inverse can be calculated with great accuracy. At the opposite, if the $I$-index is large, the matrix is considered as ill-conditioned. Some $I$-index values defining practical limits can be found in the literature (Gujarati, D.N, 1988). This a priori analysis allows to distinguish the potentially identifiable combinations $(I \leq 2)$ of material parameters from those which are not $(I>3)$. These bound values 2 and 3 are related to the shape of the objective function if an updating would be performed from the responses contained in $\overline{\mathbf{H}}^{\mathbf{p}}$. The case $I=0$ corresponds to the best conditioning, the shape of the objective function would be a $n_{\theta}$-sphere, where $n_{\theta}$ is the number of parameters. The objective function shape becomes a more general quadric surface as soon as $I>0$. If the parameters uncertainties are considered two by two, the objective function iso-value is an ellipse in the space of parametric relative uncertainties. The larger $I$, the larger the ellipticity (flattening) which means that a "valley of solutions" exists (Figure 4). The valley thinness becomes a problem for $I=2$. Considerable uncertainties can thus be generated. The bounds 2 and 3 correspond to a ratio of relative uncertainties over the estimated parameters which can reach the values 10 and 30 , respectively. Restricting to a parameters pair, the index can be graphically determinate by calculating the ratio between the minor and major radius of the smallest ellipse dimensions (Figure 4). 


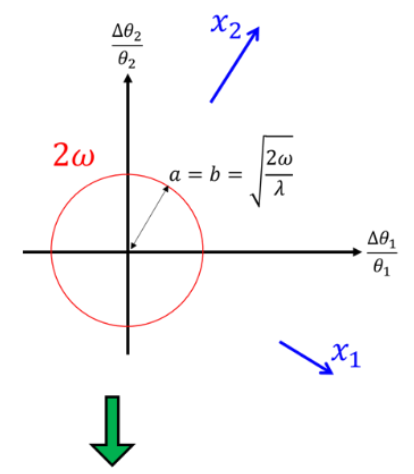

$\frac{a}{b}=1$

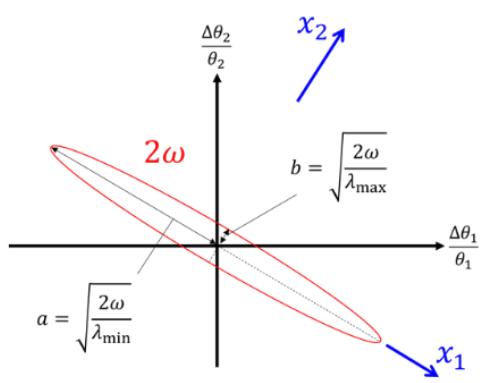

$\sqrt{2}$

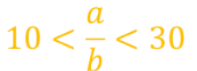

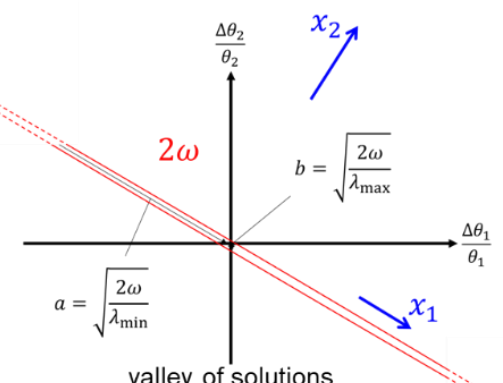

valley of solutions $\frac{a}{b}>30$

$I=3$

Critic

Figure 4 - Schematic objective function $\omega$ iso-value in the space of parametric relative uncertainties $\left(\frac{\Delta \theta_{1}}{\theta_{1}}, \frac{\Delta \theta_{2}}{\theta_{2}}\right)$ for different values of $I$-index.

The $I$-index can be calculated for all the $p$-combinations $(p=2, \ldots 9)$ of the plastic parameters, i.e. 501 possibilities.

\subsection{Method using residual topographies}

The indentation force $P(t)$ sampled in $T$ time steps $t_{k}$ may be formally replaced by the residual topography $Z(X Y)$ sampled in $M$ space steps $X Y_{k}$ along a path. The components of the $\overline{\mathbf{H}}^{\mathbf{Z}}$ matrix and the components of the sensitivity vector to parameter $\theta_{j}$ become respectively:

$$
\begin{aligned}
& \bar{H}_{i j}^{\mathbf{z}}=\sum_{k=1}^{M} \bar{S}_{k i}^{\mathbf{z}} \bar{S}_{k j}^{\mathbf{z}} \\
& \bar{S}_{k j}^{\mathbf{z}}=\frac{\theta_{j}}{Z_{\max } \sqrt{M}} \frac{\partial Z_{k}}{\partial \theta_{j}}
\end{aligned}
$$

When $n_{\mathbf{z}}$ residual topographies $\left(\mathbf{Z}^{1}, \ldots, \mathbf{Z}^{n_{\mathbf{z}}}\right)$ are combined, these expressions are written:

$$
\begin{gathered}
\bar{H}_{i j}^{\mathbf{z}^{1}, \ldots, \mathbf{z}^{n_{\mathbf{Z}}}}=\sum_{l=1}^{M \times n_{\mathbf{z}}} \bar{S}_{l i}^{\mathbf{z}^{1}, \ldots, \mathbf{z}^{n_{\mathbf{z}}}} \bar{S}_{l j}^{\mathbf{z}^{1}, \ldots, \mathbf{z}^{n_{\mathbf{z}}}} \\
\bar{S}_{l j}^{\mathbf{z}^{1}, \ldots, \mathbf{z}^{n_{\mathbf{z}}}}=\frac{\theta_{j}}{\sqrt{M}} \sum_{e=1}^{n_{\mathbf{z}}} \frac{1}{Z_{\max }^{(e)}} \sum_{k=1}^{M} \frac{\partial Z_{k}^{(e)}}{\partial \theta_{j}}
\end{gathered}
$$




\section{Sensibilities of the CPFEM to the plastic parameters}

The identifiability index is based on sensibilities of the CPFEM. As an example, in this section, the sensitivity of the simulated loading indentation curve (Eq. (8)) and residual topography (Eq. (10)) to the plastic material parameters $\boldsymbol{\theta}=\left(r_{0}, q, b, h_{1}, h_{2}, h_{3}, h_{4}, h_{5}, h_{6}\right)$ is calculated around the chosen virtual material in the [101] orientation and for a azimuth $\alpha_{[101]}^{[010]}=74^{\circ}$. Ten simulations of the nanoindentation test have been performed to approximate derivatives by the forward finite difference method:

- 1 nominal simulation using the reference parameters vector $\boldsymbol{\theta}$ (Table 2),

- 9 simulations for which one parameter $\theta_{j}$ has been successively perturbated by the quantity $\xi \theta_{j}$.

The perturbation value $\xi$ has to balance the truncation and the rounding errors performed on the simulated indentation curve and residual topography (Pottier, 2010). A stability study on both responses has shown that $\xi=10^{-3}$ is an adequate value (Renner, 2016).

\subsection{Sensitivity of the indentation curve}

In that case, the loading indentation curve $\mathbf{P}$ is the unique observation. The unloading is assumed to be purely elastic which means that no information about the plastic parameters can be extracted from this part of the curve. The measurements collected during unloading phase is thus not used. It has been noticed that the simulated loading curves were slightly disturbed due to the mesh element size, especially in the contact region. This perturbation can affect the sensitivity of indentation curve to the material parameters and thus the identifiability calculations. To avoid any problems, the simulated loading curves $P(h)$ have been fitted by a third-order polynomial. The purpose here is not to define the relationship between the indenter force and displacement, but to filter out any perturbation due to the mesh element size.

The simulated indentation loading curves are sampled in $T=181$ time steps. The sensitivity of the simulated indentation force to the parameters is defined using Eq. (8). This definition attributes a small weight to small indentation depth. Thus, the contact detection problem is limited for the sensitivity calculation at small indentation depth. The Figure 5 presents the obtained sensitivity vectors and their norms. The indentation curves are sensitive to all parameters and their norm are of comparable in magnitude. The curve is more sensitive to $r_{0}$, $q, b$ and $h_{3}$ than to the other parameters. The ratio between the sensitivities to the parameter $b$ (the most influent) and $h_{2}$ (the less influent) is about 4.2.

The collinearity of some sensitivity vectors to some parameters is highlighted in Figure $5 \mathrm{a}$. It is an extremely important measurement since the inversion problem is impossible when sensitivity vectors are colinear. One can notice that the sensitivity vectors associated to the 
parameter pairs $\left(r_{0}, q\right),\left(b, h_{3}\right),\left(h_{1}, h_{4}\right)$ and $\left(h_{1}, h_{6}\right)$ seem to be almost collinear. At this stage, difficulties in the parametric identification from the sole indentation curve can be anticipated.

(a)

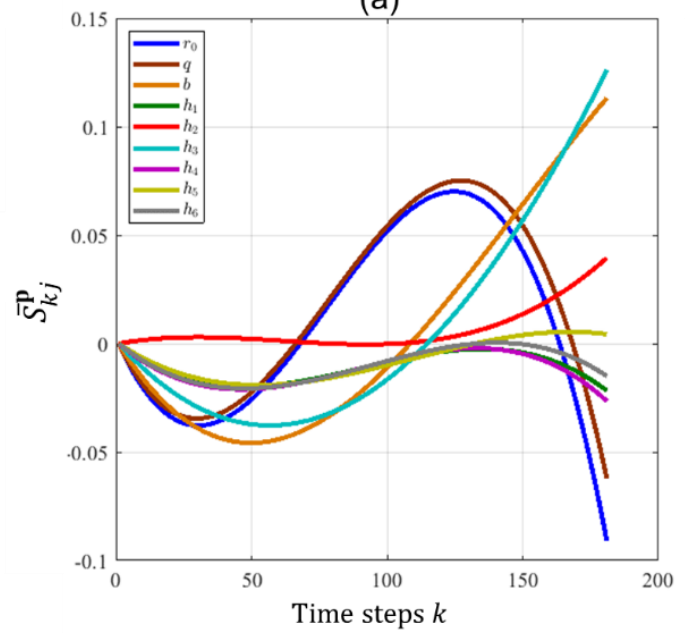

(b)

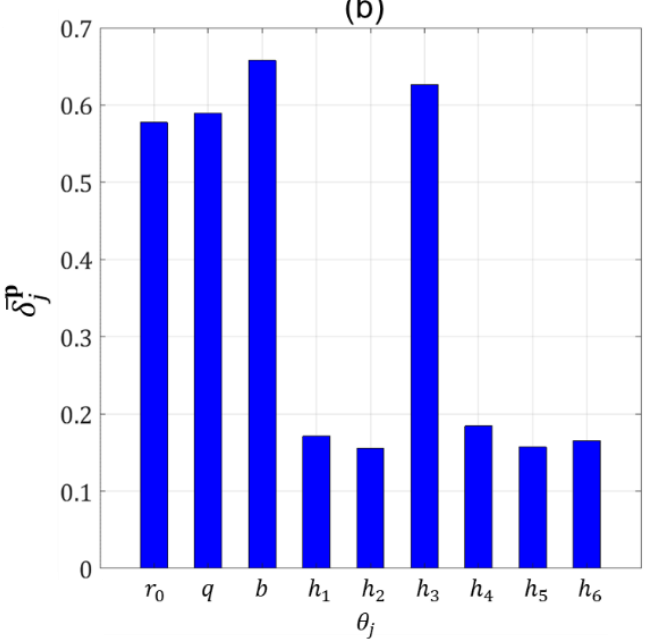

Figure 5 - Sensitivity of the loading indentation curve $\mathbf{P}$ to the parameters of the virtual material: (a) sensitivity vectors $\bar{S}_{k j}^{\mathbf{P}}$ and (b) norms $\bar{\delta}_{j}^{\mathbf{P}}=\sqrt{\bar{S}_{k j}^{\mathbf{P}} \bar{S}_{k j}^{\mathbf{P}}}$.

\subsection{Sensitivity of the residual topography}

In that case, the residual topography $\mathbf{Z}$ obtained in the [101] orientation and for an azimuth $\alpha_{[101]}^{[010]}=74^{\circ}$ is the unique observation. Initially, topography data are $256 \times 256$ matrices, which corresponds to the picture definition $\left(256 \times 256 \mathrm{pix}^{2}\right)$. Topography data are reshaped in a 65536-components vector along the path illustrated in (Figure 6a). The experimental AFM measurements of the imprint centre is poorly defined. Indeed, the bottom of the imprint cannot be well-measured since the AFM is used in non-contact mode. The measurements collected close to the imprint centre should therefore not be used. 

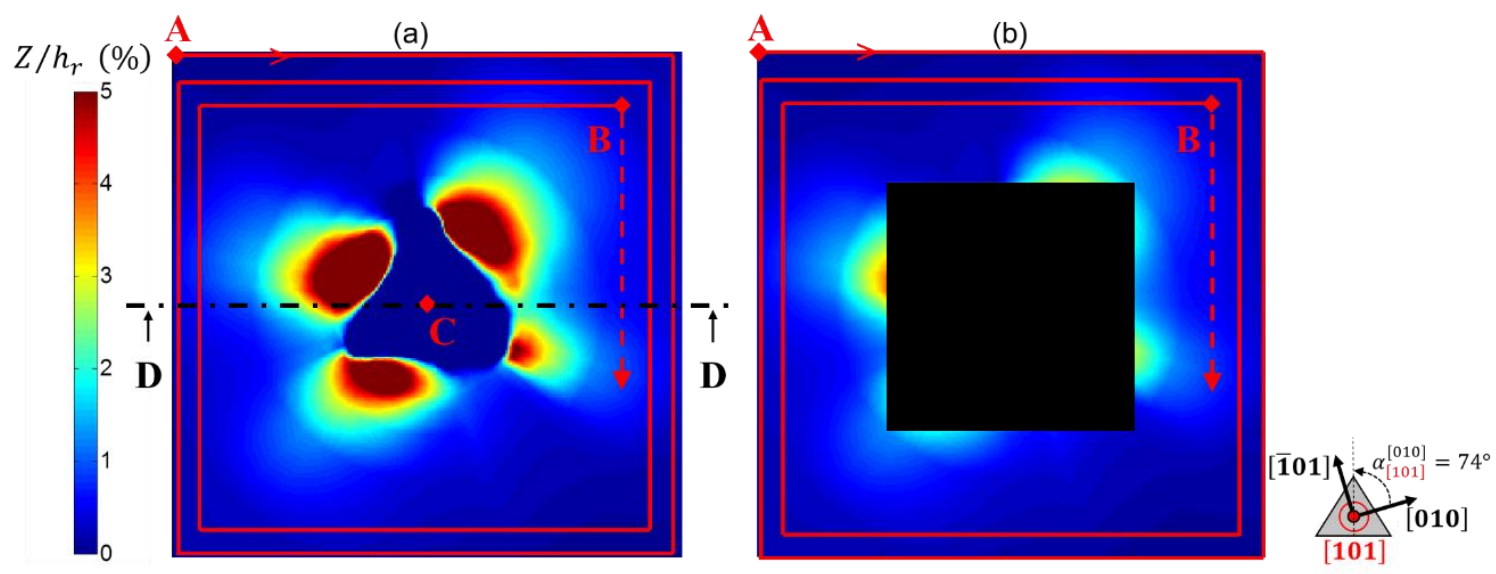

Figure 6 - (a) Topography $\mathbf{Z}=\mathbf{Z}^{1}$ data reshaping from square matrix to vector form along the red path. $\mathbf{A}$ is the starting point and $\mathbf{C}$ is the end point (topography centre). (b) $125 \times 125$ pix $^{2}$ square truncation of the topography.

To limit the poor definition effects of the imprint bottom, the data are slightly changed to assign a small weight to the bottom of the imprint and a maximum weight to pile-ups:

$$
Z=1+\frac{Z_{0}}{h_{r}}
$$

where $Z_{0}$ and $h_{r}$ are the initial residual topography and the residual indentation depth (Figure $3)$. The zero of $Z_{0}$ is the sample surface far from the imprint. Using this definition, the bottom of the imprint is the zero of the observation (Figure 7).

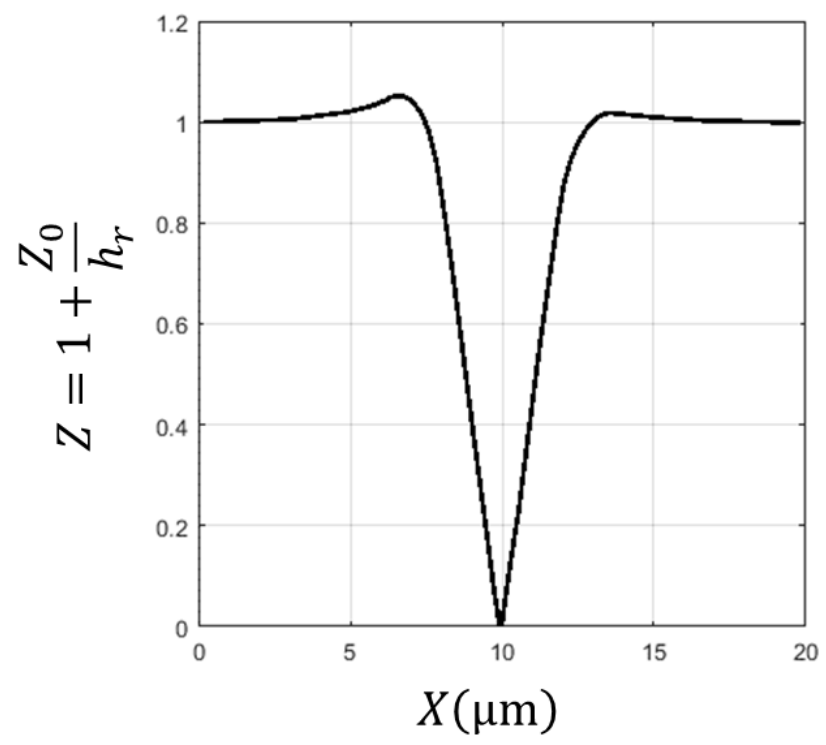

Figure 7 - Profile of the topography $\mathbf{Z}=\mathbf{Z}^{1}$ along the D-D cut (Figure 6). 
It is also chosen to perform a square shape truncation of the information at the imprint centre (Figure 6b). A smart truncation decreases the measurements and avoids the use of data disturbed by the contact tip-sample. In that case $\left(125 \times 125 \mathrm{pix}^{2}\right)$, the imprint centre is then entirely covered and the topography is sampled at about $M=5 \times 10^{4}$ positions. The sensitivity of the simulated imprint topography to the parameters is defined using Eq. (10) and is presented in Figure 8ab. The same quantities are shown in Figure 8cd using the truncated topography presented in Figure 6b. The sensitivity vectors norms are extremely affected. Contrarily, the relative parameters influence on the topography remains unchanged, regardless to the truncation size.

The residual topography is more sensitive to $r_{0}, q, b$ and $h_{3}$ compared to the other parameters in both cases. The ratio between the highest sensibility and the lowest $\left(h_{2}\right)$ is about 7.7 if the topography is complete and about 12.6 using the truncation, which already predicts identification problems.

(a)

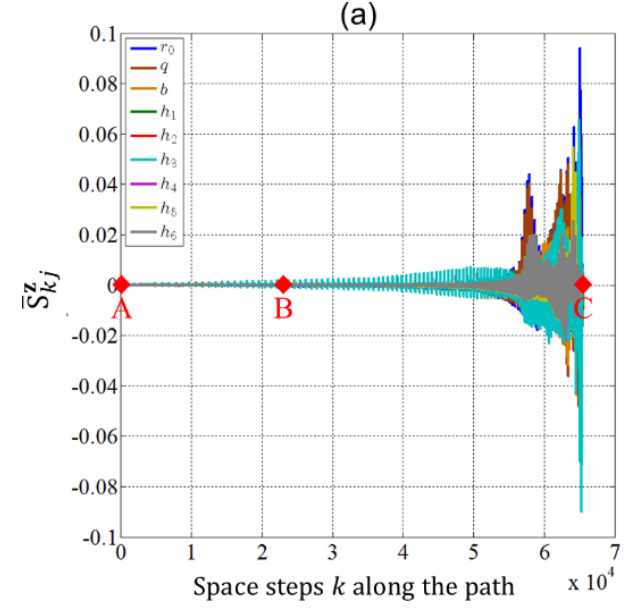

(c)

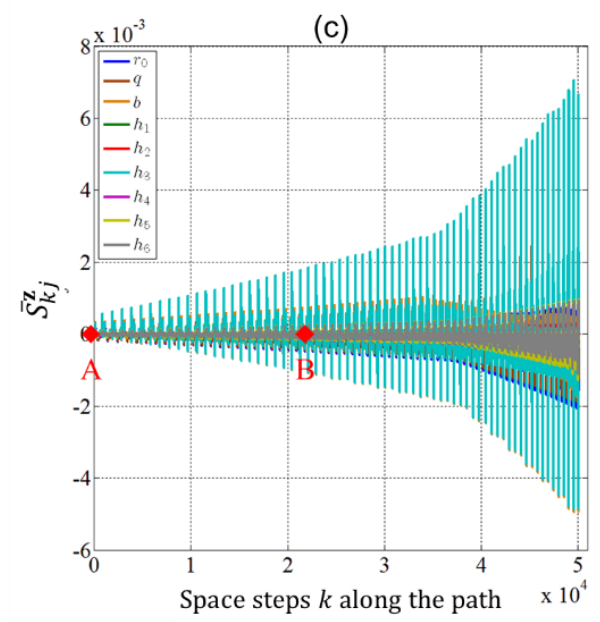

(b)

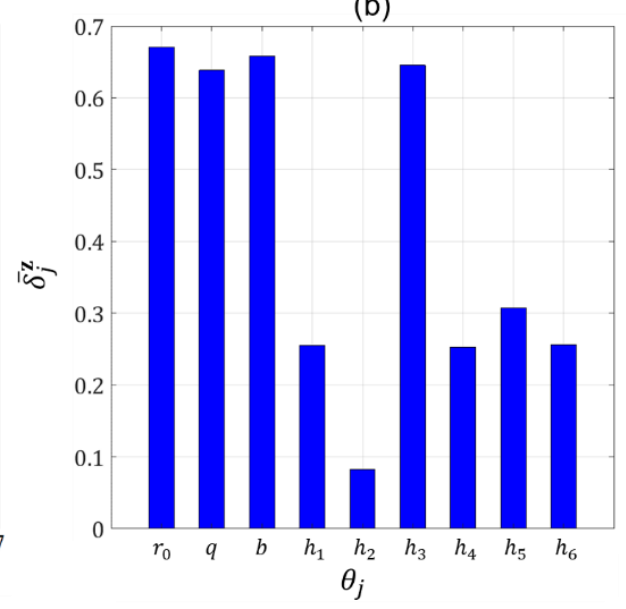

(d)

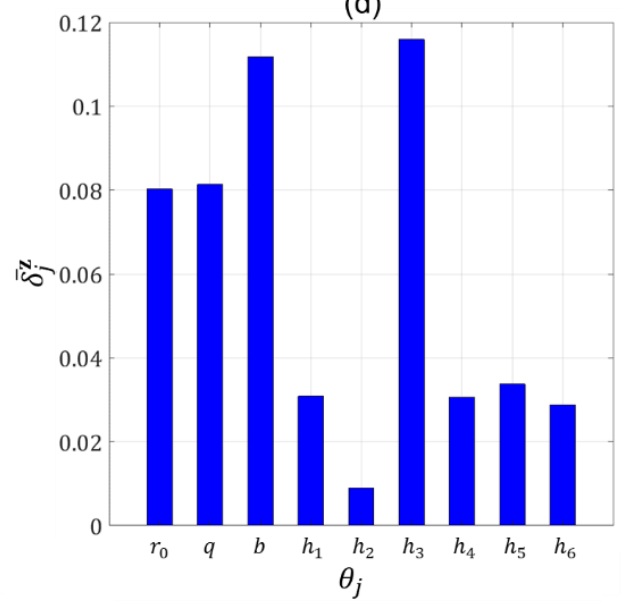

Figure 8 - Entire imprint topography $\mathbf{Z}=\mathbf{Z}^{1}$ : (a) sensitivity vectors $\bar{S}_{k j}^{\mathbf{Z}}$ and (b) norms $\bar{\delta}_{j}^{\mathrm{z}}=\sqrt{\bar{S}_{k j}^{\mathrm{z}} \bar{S}_{k j}^{\mathrm{z}}}$. Truncated imprint: (c) sensitivity vectors and (d) norms. 
One can notice that the calculation of the sensitivity can be largely affected by the information quantity. Having a large volume of information does not mean that the post processing will be of good quality.

\section{Identifiability results}

The section 4 highlighted the problems of sensitivity vectors multi-collinearity and the gap between norms. Both aspects are taken into account with the use of the identifiability index. In the following sections, the identifiability of the plastic parameters $\left(r_{0}, q, b, h_{1}, h_{2}, h_{3}, h_{4}, h_{5}, h_{6}\right)$ has been calculated in several cases:

- using one indentation curve $\mathbf{P}\left([101], \alpha_{[101]}^{[010]}=74^{\circ}\right)$, the same as the one studied in section 4.1 ,

- $\quad$ using one topography $\mathbf{Z}^{1}=\mathbf{Z}\left([101], \alpha_{[101]}^{[010]}=74^{\circ}\right)$, the same as the one studied in section 4.2 ,

- $\quad$ using a combination of two topographies: $\mathbf{Z}^{1}$ and $\mathbf{Z}^{2}\left([001], \alpha_{[001]}^{[100]}=77^{\circ}\right)$,

- $\quad$ using a combination of three topographies: $\mathbf{Z}^{1}, \mathbf{Z}^{2}$ and $\mathbf{Z}^{3}\left([111], \alpha_{[111]}^{[\overline{1} 2 \overline{1}]}=65^{\circ}\right)$.

It is recalled that all simulated responses are the results of the nanoindentation CPFEM around the virtual material defined in section 2.2.

\subsection{Results using one indentation curve}

As expected, when all parameters are considered for the index calculation $I_{\mathbf{p}}>6$. It means that the identification of all parameters using the sole indentation curve is impossible. $I_{\mathbf{P}}$ has also been calculated for all pairs of parameters. The results are presented in Table 3. All the pairs which have an identifiability index $I_{\mathbf{p}} \leq 2$ are a priori identifiable using $\mathbf{P}$, assuming the other parameters are known. This is true only if the material behaviour to identify is close to the virtual material. For the pairs $\left(b, h_{3}\right)$ and $\left(h_{1}, h_{4}\right), I_{\mathbf{p}}>2$, so that the identification of even 2 parameters can remain difficult using this indentation curve. The result for the pairs $\left(r_{0}, q\right)$ and $\left(h_{1}, h_{6}\right)$ are only slightly better. These results been speculated from the sensitivity analysis in section 4.1 (Figure 5a): for these pairs, the sensitivity vectors to each parameter are almost collinear. 


\begin{tabular}{|c|c|c|c|c|c|c|c|c|c|}
\hline$I_{\mathbf{p}}$ & $r_{0}$ & $q$ & $b$ & $h_{1}$ & $h_{2}$ & $h_{3}$ & $h_{4}$ & $h_{5}$ & $h_{6}$ \\
\hline$r_{0}$ & & 1.9 & 0.2 & 1.1 & 1.1 & 0.1 & 1.0 & 1.1 & 1.1 \\
\hline$q$ & & & 0.3 & 1.1 & 1.2 & 0.2 & 1.0 & 1.1 & 1.1 \\
\hline$b$ & & & & 1.2 & 1.7 & 2.3 & 1.1 & 1.5 & 1.2 \\
\hline$h_{1}$ & & & & & 0.5 & 1.1 & 2.9 & 1.0 & 2.0 \\
\hline$h_{2}$ & & & & & & 1.8 & 0.5 & 0.1 & 0.3 \\
\hline$h_{3}$ & & & & & & & 1.1 & 1.4 & 1.2 \\
\hline$h_{4}$ & & & & & & & & 0.9 & 1.8 \\
\hline$h_{5}$ & & & & & & & & & 1.3 \\
\hline$h_{6}$ & & & & & & & & & \\
\hline
\end{tabular}

Table 3 - I-index for all the parameters pairs using the indentation curve $\mathbf{P}$.

$I_{\mathbf{p}} \leq 2$ (green, potentially identifiable), $2<I_{\mathbf{p}} \leq 3$ (orange, difficult to identify), $I_{\mathbf{p}}>3$ (red, not identifiable).

In the case where the interaction matrix components $\left(h_{1}, h_{2}, h_{3}, h_{4}, h_{5}, h_{6}\right)$ are known, $I_{\mathbf{p}}\left(r_{0}, q, b\right)=2.3$. The identification of the isotropic hardening parameters is thus difficult. These results are in line with those in the literature at macroscopic scale. For a macroscopic power hardening law using 2 plastic parameters (yield stress $\sigma_{y}$ and exponent hardening $n$ ), authors generally conclude that it is impossible to obtained a stable solution for the pair $\left(\sigma_{y}, n\right)$ using a conical indentation curve (Alkorta et al., 2005; Capehart and Cheng, 2003; Casals and Alcalá, 2005; Charleux, 2006; Tho et al., 2004).

In the opposite case, where the isotropic hardening parameters $\left(r_{0}, q, b\right)$ are known, $I_{\mathbf{p}}\left(h_{1}, h_{2}, h_{3}, h_{4}, h_{5}, h_{6}\right)>6$. Thus, the interaction matrix is not identifiable using the indentation curve $\mathbf{P}$.

\subsection{Results using one imprint}

Following the same process, the identifiability of all parameters is calculated using the truncated residual topography $\mathbf{Z}=\mathbf{Z}^{1}$ only (Figure $6 \mathrm{~b}$ ). The evolution of $I_{\mathbf{Z}^{1}}$-index as a function of the number of considered pixels in topography $\mathbf{Z}^{1}$ is presented Figure 9 . The final value tends to $I_{\mathbf{Z}^{1}}=3.6$, which means that the identification of all parameters using $\mathbf{Z}^{1}$ only is impossible. However, $\mathbf{Z}^{1}$ contains more information than the indentation curve $\left(I_{\mathbf{p}}>6\right)$. In the case where the interaction matrix components $\left(h_{1}, h_{2}, h_{3}, h_{4}, h_{5}, h_{6}\right)$ are known, $I_{\mathbf{z}}\left(r_{0}, q, b\right)=1.9$, a better value than when using the indentation curve $\left(I_{\mathbf{p}}=2.3\right)$. The identification of the isotropic hardening parameters can be envisaged and could be calculated using the tensile curves. 
In the opposite case, where the isotropic hardening parameters $\left(r_{0}, q, b\right)$ are known, $I_{\mathbf{z}}\left(h_{1}, h_{2}, h_{3}, h_{4}, h_{5}, h_{6}\right)=3.2$. Thus, the interaction matrix is not identifiable using $\mathbf{Z}^{1}$.

In all three cases, the pile-ups affect the index evolution, it changes the slope around $k=4.10^{4}$ (Figure 9) and tends to decrease the index values. It means that pile-ups are rich in information in that case.

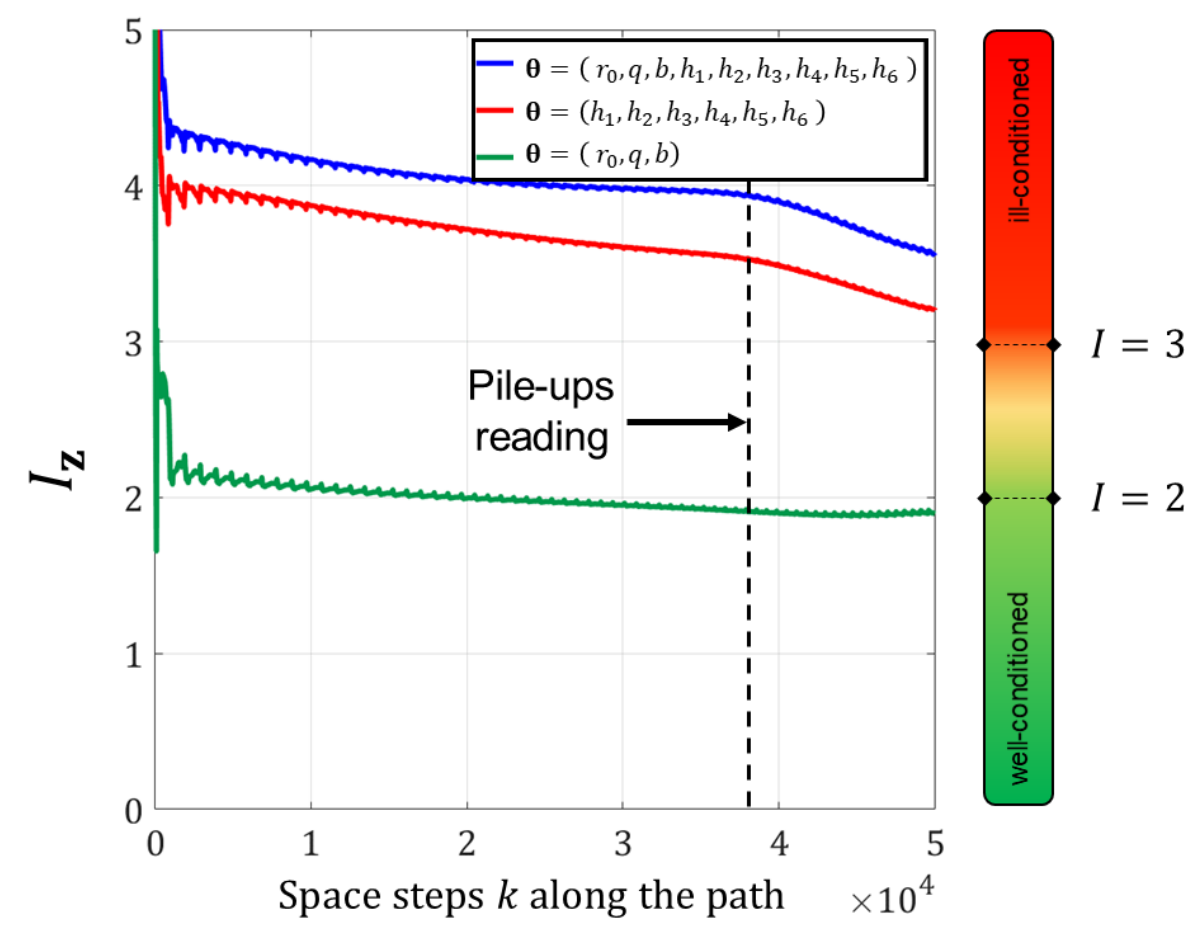

Figure $9-I_{\mathbf{z}}$ evolution over the topography $\mathbf{Z}=\mathbf{Z}^{1}$ for: all parameters (blue), isotropic hardening parameters $\left(r_{0}, q, b\right)$ only (green) and interaction matrix components $\left(h_{1}, h_{2}, h_{3}, h_{4}, h_{5}, h_{6}\right)$ only (red).

As in the previous section, $I_{\mathbf{z}^{1}}$ has also been calculated for all pairs of parameters (Table 4). One can notice that $I_{\mathbf{z}^{1}}>2$ for $\left(b, h_{2}\right),\left(b, h_{3}\right),\left(h_{1}, h_{4}\right),\left(h_{1}, h_{6}\right)$ and $\left(h_{2}, h_{3}\right)$, which means that the identification of 2 parameters can also remain difficult using this imprint topography. 


\begin{tabular}{c|ccccccccc}
\hline$I_{\mathbf{z}^{1}}$ & $r_{0}$ & $q$ & $b$ & $h_{1}$ & $h_{2}$ & $h_{3}$ & $h_{4}$ & $h_{5}$ & $h_{6}$ \\
\hline$r_{0}$ & & 1.8 & 0.3 & 0.9 & 1.9 & 0.4 & 1.0 & 0.9 & 1.0 \\
$q$ & & & 0.3 & 1.0 & 1.9 & 0.3 & 1.1 & 1.0 & 1.1 \\
$b$ & & & & 1.2 & 2.2 & 2.4 & 1.2 & 1.1 & 1.3 \\
$h_{1}$ & & & & & 1.1 & 1.2 & 2.3 & 2.0 & 2.1 \\
$h_{2}$ & & & & & & 2.2 & 1.1 & 1.2 & 1.0 \\
$h_{3}$ & & & & & & & 1.2 & 1.2 & 1.3 \\
$h_{4}$ & & & & & & & & 1.9 & 2.0 \\
$h_{5}$ & & & & & & & & & \\
$h_{6}$ & & & & & & & & & \\
\hline
\end{tabular}

Table $4-I_{\mathbf{z}^{1}}$-index for all the parameters pairs using the residual topography $\mathbf{Z}^{1}: I_{\mathbf{z}^{1}} \leq 2$ (green, potentially identifiable), $2<I_{\mathbf{z}^{1}} \leq 3$ (orange, difficult to identify), $I_{\mathbf{z}^{1}}>3$ (red, not identifiable).

These results agree with the Bolzon et al. works conducted on elastic-plastic indentations using a macroscopic power law hardening (Bolzon et al., 2011). They concluded that the imprint mapping is a richer observation than the indentation curve and the $l$-index calculated graphically on Bolzon's results supports this conclusion (Figure 10).

(a)

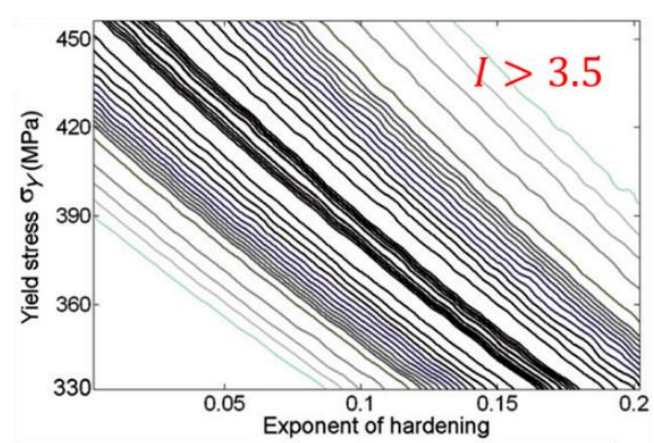

(b)

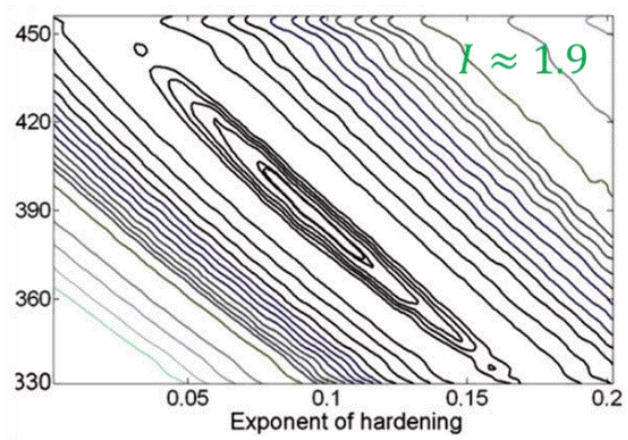

Figure 10 - Objective functions iso-values obtained by Bolzon et al. using macroscopic FEM

(Bolzon et al., 2011): (a) indentation curve only $I>3.5$, (b) residual profile only $I \approx 1.9$. $I$-index values graphically estimated.

\subsection{Results using combinations of imprints}

\subsubsection{Combination of two topographies}

The identifiability of parameters is now calculated using two truncated residual topographies $\mathbf{Z}^{1}$ and $\mathbf{Z}^{2}$ obtained in the [001] orientation and $\alpha_{[001]}^{[100]}=77^{\circ}$ (Figure 11). 

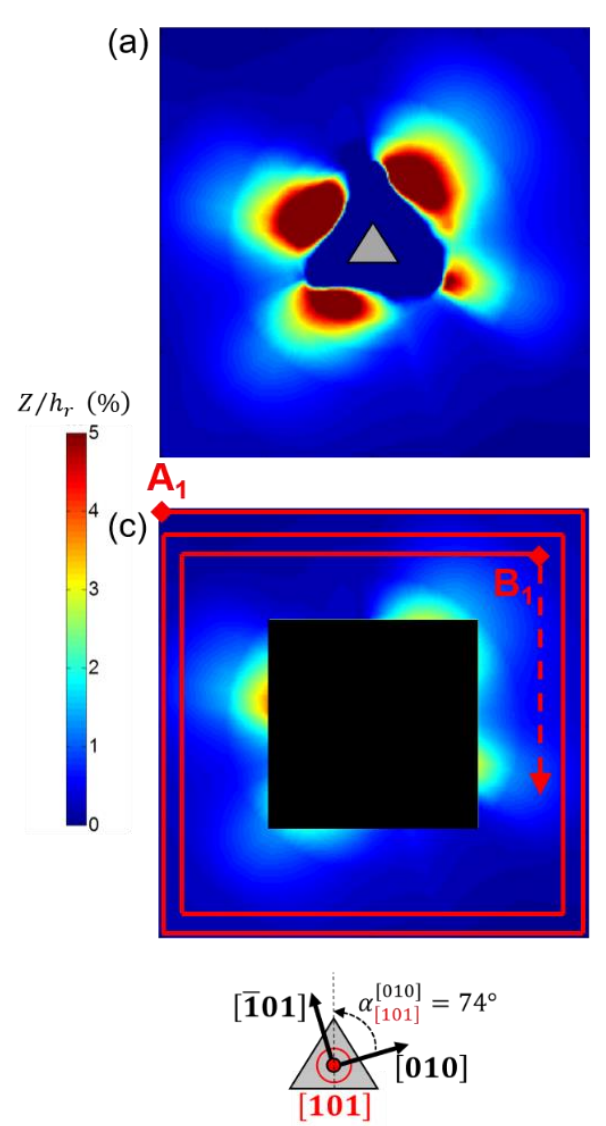

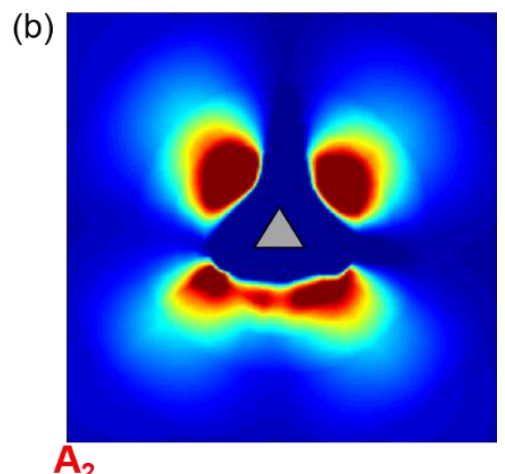

(d)
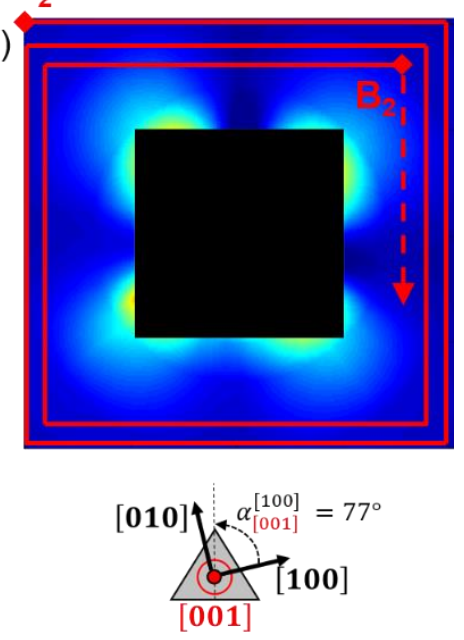

Figure 11 -Topography $\mathbf{Z}=\mathbf{Z}^{1}$ : (a) complete and (c) truncated; Topography $\mathbf{Z}^{2}$ : (b) complete and (d) truncated. $\mathbf{A}_{1}, \mathbf{B}_{1}, \mathbf{A}_{2}$ and $\mathbf{B}_{2}$ are specific points.

Overall, in Figure 12, the sensitivity of the imprint topography $\mathbf{Z}^{2}$ to the parameters is significantly lower than for the imprint topography $\mathbf{Z}^{1}$. Except for the sensitivity to the parameters $h_{5}$ and $h_{6}$ for which the norm values almost doubled, the norm values are slightly higher if both topographies are taken into account (Figure 13). The ratio between the sensitivity to the parameter $h_{3}$ (the most influent) and $h_{2}$ (the less influent) still remain very large. 


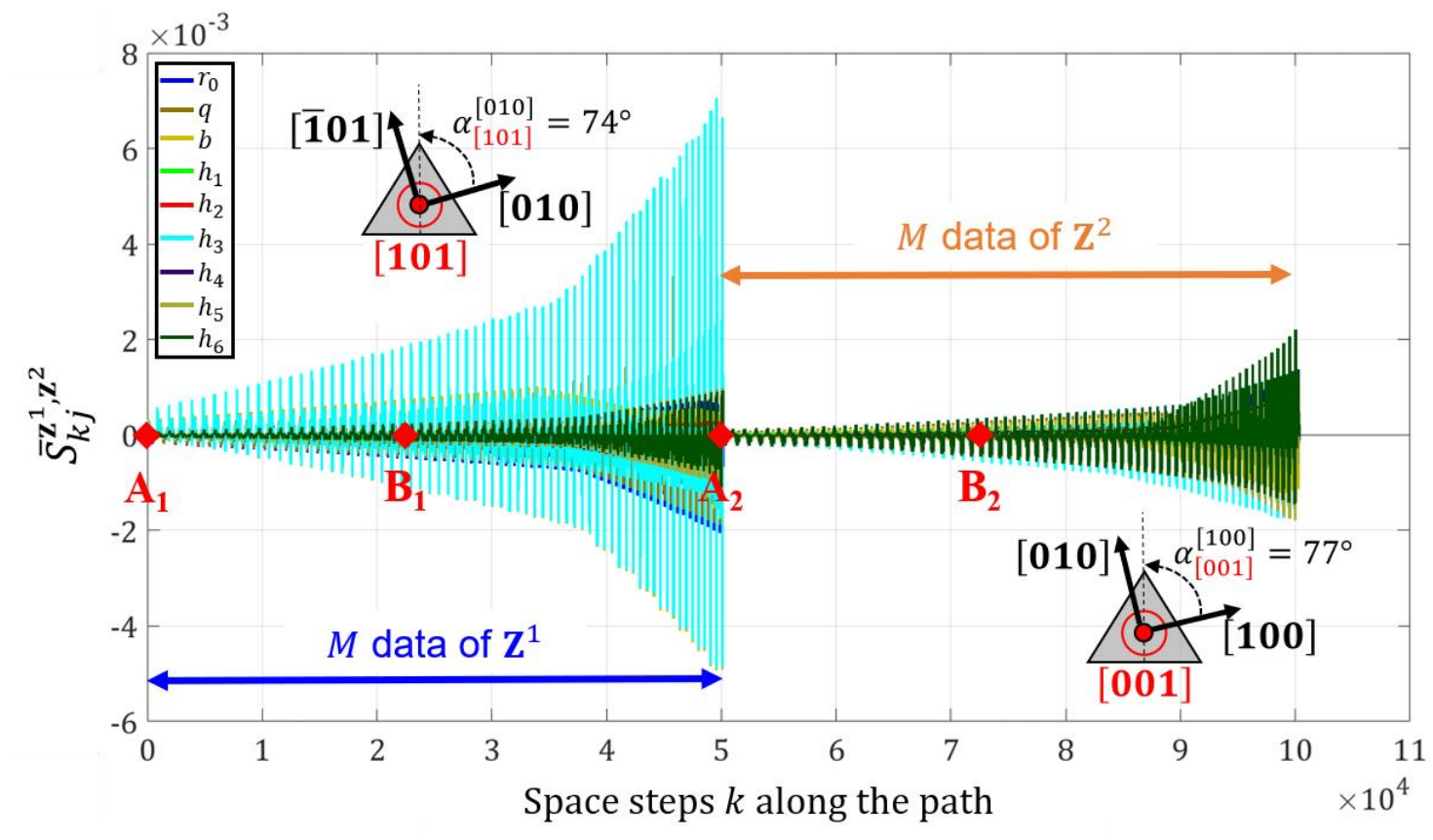

Figure 12 - Sensitivity vectors of both truncated topographies $\mathbf{Z}^{1}$ and $\mathbf{Z}^{2}$ to each parameter. $\mathbf{A}_{1}, \mathbf{B}_{1}, \mathbf{A}_{2}$ and $\mathbf{B}_{2}$ are specific topography points.

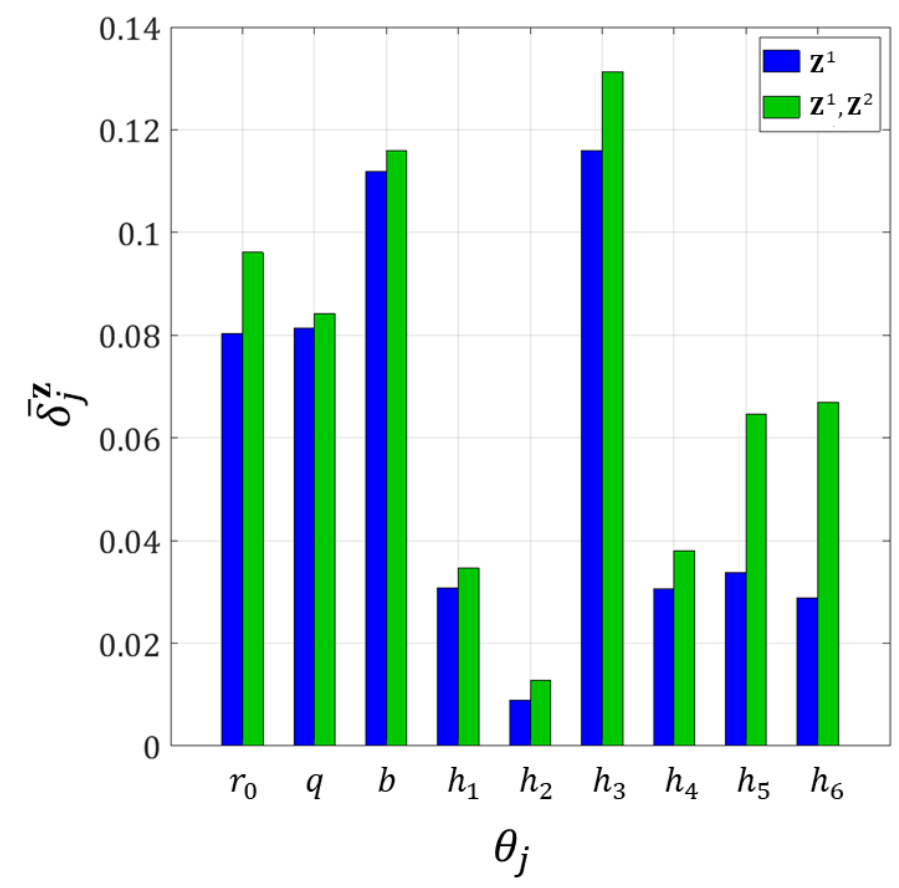

Figure 13 - Norm of the simulated topographies sensitivity to each parameter: $\mathbf{Z}^{1}$ (blue) and combination $\mathbf{Z}^{1}, \mathbf{Z}^{2}$ (green).

The Table 5 presents the calculated values of the $I_{\mathbf{z}^{1}, \mathbf{z}^{2}}$-index (lower part) for all parameters pairs and are compared to those of $I_{\mathbf{z}^{1}}$ (upper part). One can notice that all pairs are identifiable when the second topography $\mathbf{Z}^{2}$ is added in the process. It means that the non-collinearities 
are increased if $\mathbf{Z}^{2}$ is added to the process, even if the vectors norms are not of the same order of magnitude.

\begin{tabular}{c|ccccccccc}
\hline$I_{\mathbf{z}^{1}}$ & & & & & & & & & \\
$I_{\mathbf{z}^{1}, \mathbf{z}^{2}}$ & $r_{0}$ & $q$ & $b$ & $h_{1}$ & $h_{2}$ & $h_{3}$ & $h_{4}$ & $h_{5}$ & $h_{6}$ \\
\hline$r_{0}$ & & 1.8 & 0.3 & 1.0 & 1.9 & 0.4 & 1.0 & 0.9 & 1.0 \\
$q$ & 0.9 & & 0.3 & 1.0 & 1.9 & 0.3 & 1.1 & 1.0 & 1.1 \\
$b$ & 0.2 & 0.3 & & 1.2 & 2.2 & 2.4 & 1.2 & 1.1 & 1.3 \\
$h_{1}$ & 1.0 & 0.9 & 1.1 & & 1.1 & 1.2 & 2.3 & 2.0 & 2.1 \\
$h_{2}$ & 1.8 & 1.6 & 1.9 & 0.9 & & 2.2 & 1.1 & 1.2 & 1.0 \\
$h_{3}$ & 0.3 & 0.4 & 1.1 & 1.2 & 2.0 & & 1.2 & 1.2 & 1.3 \\
$h_{4}$ & 0.9 & 0.9 & 1.0 & 1.0 & 1.0 & 1.1 & & 1.9 & 2.0 \\
$h_{5}$ & 0.7 & 0.3 & 0.5 & 0.7 & 1.4 & 0.9 & 0.7 & & 2.0 \\
$h_{6}$ & 0.7 & 0.3 & 0.5 & 0.7 & 1.4 & 0.8 & 0.7 & 1.1 & \\
\hline
\end{tabular}

Table 5 - $I$-index for all the parameter pairs using one $\left(\mathbf{Z}^{1}\right)$ and two $\left(\mathbf{Z}^{1}, \mathbf{Z}^{2}\right)$ residual topographies: $I \leq 2$ (green, potentially identifiable), $2<I \leq 3$ (orange, difficult to identify), $I>$ 3 (red, not identifiable).

As in the previous sections, the $\mathrm{I}_{\mathbf{Z}^{1}, \mathbf{Z}^{2}}$-index is calculated in three cases for $\left(r_{0}, q, b, h_{1}, h_{2}, h_{3}, h_{4}, h_{5}, h_{6}\right),\left(r_{0}, q, b\right)$ and $\left(h_{1}, h_{2}, h_{3}, h_{4}, h_{5}, h_{6}\right)$. In all three cases, using both topographies $\mathbf{Z}^{1}$ and $\mathbf{Z}^{2}$ reduces the index values (Figure 14).

For $\left(r_{0}, q, b, h_{1}, h_{2}, h_{3}, h_{4}, h_{5}, h_{6}\right)$, the index value tends to $I_{\mathbf{Z}^{1}}, \mathbf{Z}^{2}=2.4$, which is better than using the first topography $\mathbf{Z}^{1}$ only $\left(I_{\mathbf{Z}^{1}}=3.6\right)$. It means that the identification of all parameters $\boldsymbol{\theta}$ using both topographies is not impossible but remains difficult.

In the case where the interaction matrix components $\left(h_{1}, h_{2}, h_{3}, h_{4}, h_{5}, h_{6}\right)$ are known, $I_{\mathbf{Z}^{1}, \mathbf{Z}^{2}}\left(r_{0}, q, b\right)=0.9$, which is a good value. That ensures that the identification of the isotropic hardening parameters is possible if the material behaviour to identify is very close to the virtual material and all the other parameters are known.

In the opposite case, where the isotropic hardening parameters $\left(r_{0}, q, b\right)$ are known, $I_{\mathbf{Z}^{1}, \mathbf{Z}^{2}}\left(h_{1}, h_{2}, h_{3}, h_{4}, h_{5}, h_{6}\right)=2.2$. The identifiability of the interaction matrix is significantly improved using the second topography $\mathbf{Z}^{2}\left(I_{\mathbf{Z}^{1}}=3.2\right)$.

In all three cases, the pile-ups affect the index evolution, it changes the slope around $k=4.10^{4}$ and around $k=9.10^{4}$ and tends to decrease the index values (Figure 14). It highlights the 
information contained in pile-ups. As a conclusion, the inverse problem stability is thus significantly improved when using both residual topographies $\mathbf{Z}^{1}$ and $\mathbf{Z}^{2}$.

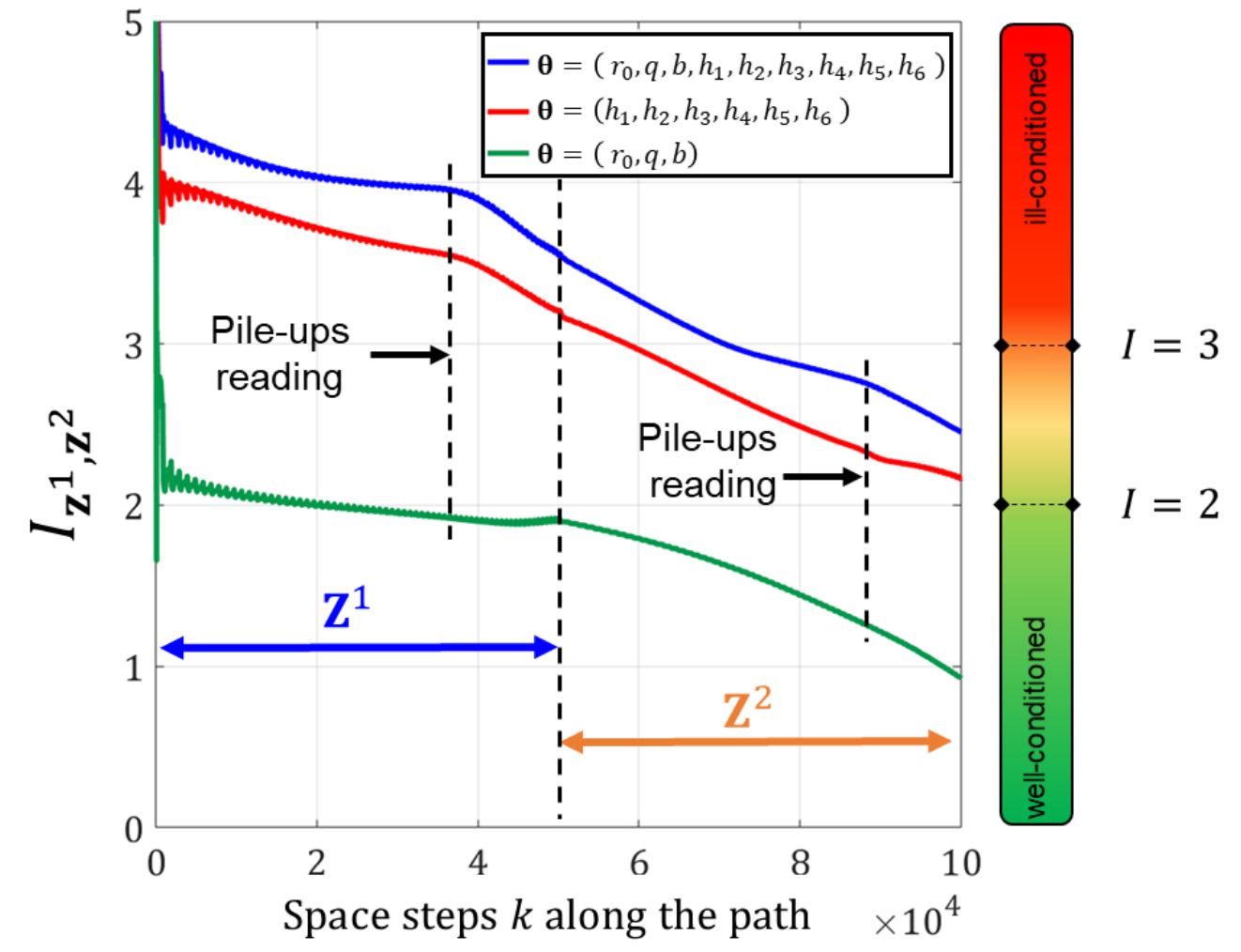

Figure $14-I_{\mathbf{z}^{1}, \mathbf{z}^{2}}$ evolution over both topographies $\mathbf{Z}^{1}$ and $\mathbf{Z}^{2}$ for: all parameters (blue), isotropic hardening parameters $\left(r_{0}, q, b\right)$ only (green) and the interaction matrix components $\left(h_{1}, h_{2}, h_{3}, h_{4}, h_{5}, h_{6}\right)$ only (red).

\subsubsection{Combination of three topographies}

In this last section, three truncated residual topographies $\mathbf{Z}^{1}, \mathbf{Z}^{2}$ and $\mathbf{Z}^{3}$ obtained in the [111] orientation and $\alpha_{[111]}^{[\overline{12} \overline{1}]}=65^{\circ}$ have been used for calculating the parameters identifiability index (Figure 15). We can notice in this figure that pile-up sizes and distributions in semi-distant field show significant asymmetries. These asymmetries result from the convolution of the crystal slip system symmetry on the considered indented direction with the three-fold Berkovich indenter symmetry. Indeed, slip systems in the [101] indentation direction feature a two-fold symmetry, while those in the [001] indentation direction display a four-fold symmetry and a sixfold (quasi rotational) symmetry in the [111] indentation direction. 

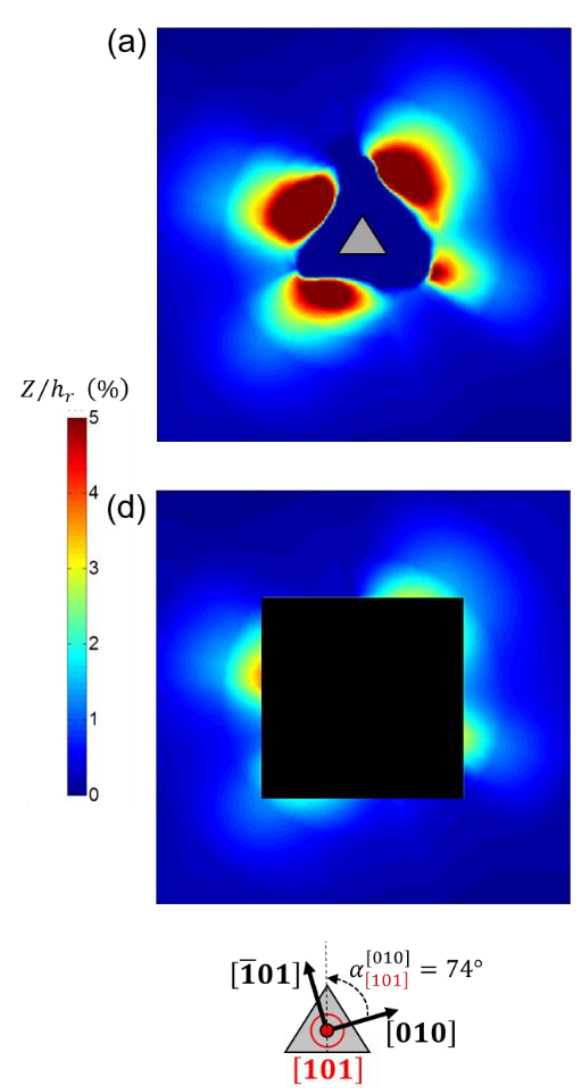

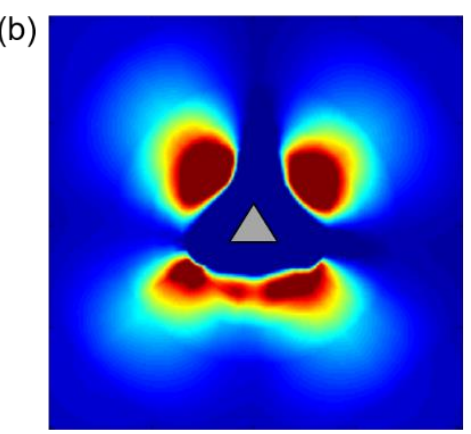

(e)
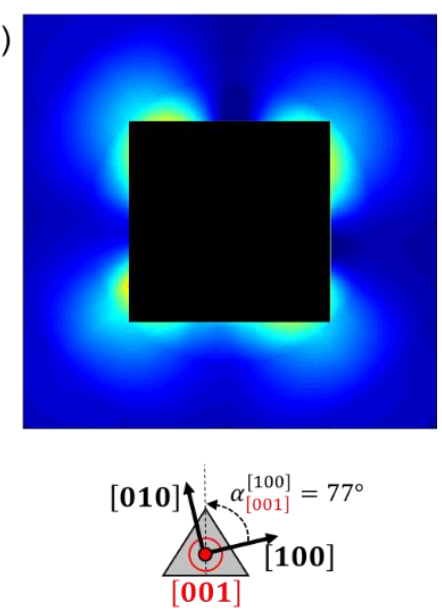

(c)

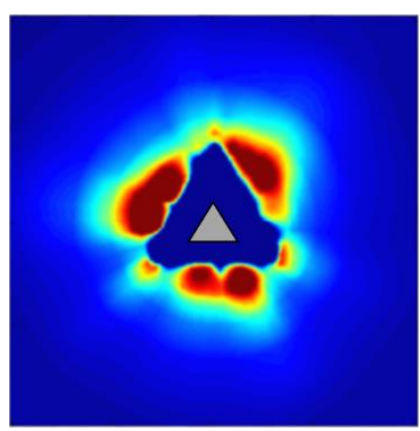

(f)
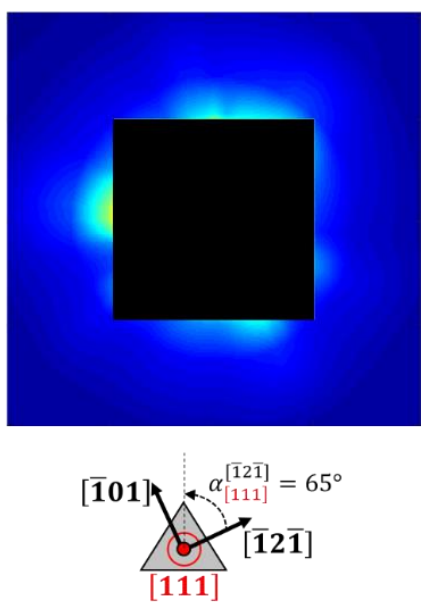

Figure 15 -Topography $\mathbf{Z}^{1}$ : (a) complete and (d) truncated; Topography $\mathbf{Z}^{2}$ : (b) complete and (e) truncated. Topography $\mathbf{Z}^{3}$ : (c) complete and (f) truncated.

In Figure 16, one can notice that the sensitivity vectors norms are significantly affected using the three imprint topographies. All norms are now the same order of magnitude which is a first sign of inverse problem conditioning improvement. The sensitivity vector norm associated to the parameter $h_{2}$ is no longer the lowest one. 


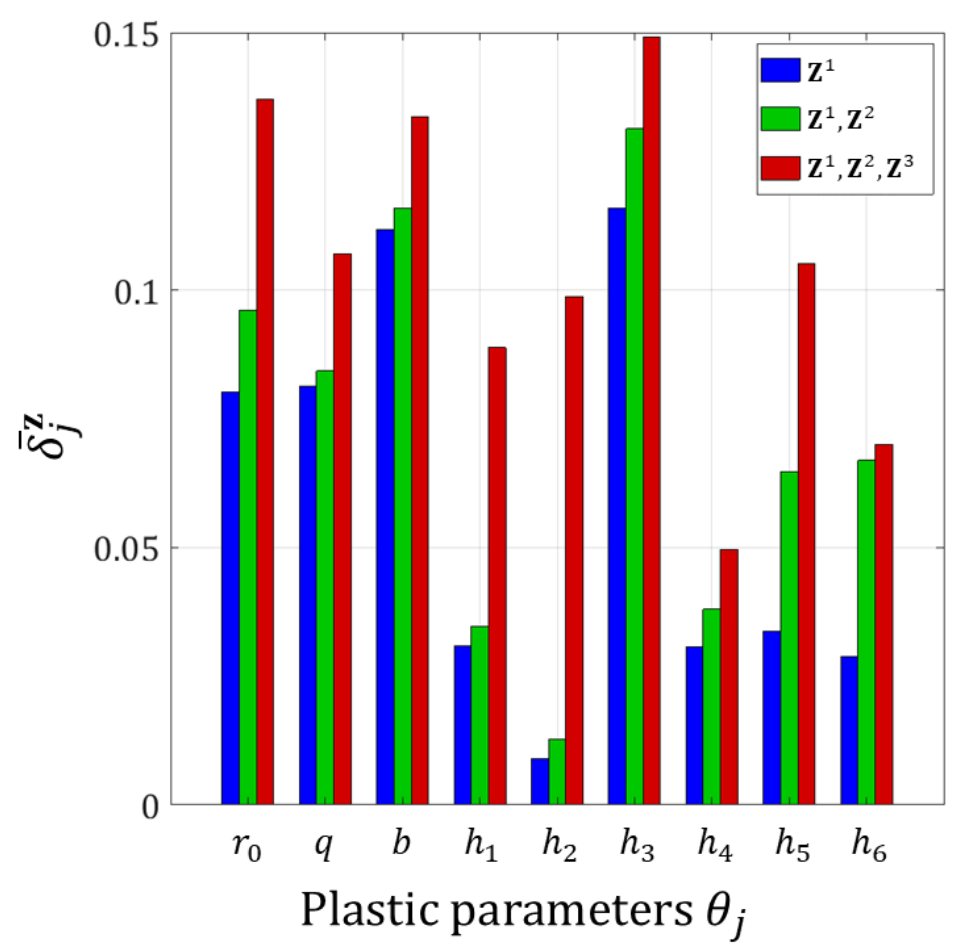

Figure 16 - Norm of the simulated topographies sensitivity to each parameter: $\mathbf{Z}^{1}$ (blue), $\mathbf{Z}^{1}, \mathbf{Z}^{2}$ (green), $\mathbf{Z}^{1}, \mathbf{Z}^{2}, \mathbf{Z}^{3}$ (red).

The $I_{\mathbf{z}^{1}, \mathbf{z}^{2}, \mathbf{z}^{3}}$-index has been calculated for all parameters pairs and compared to those of $I_{\mathbf{Z}^{1}, \mathbf{Z}^{2}}$ (Table 6). For all pairs, $I_{\mathbf{z}^{1}, \mathbf{z}^{2}, \mathbf{z}^{3}} \leq 1.2$ which predicts a good conditioning of the inverse problem for identification. Even if $I_{\mathbf{z}^{1}, \mathbf{z}^{2}, \mathbf{z}^{3}}$-index is better than using two topographies $\left(I_{\mathbf{z}^{1}, \mathbf{z}^{2}} \leq 2.0\right)$, it is interesting to notice that it has slightly increased for some pairs $\left(r_{0}, q\right),\left(r_{0}, h_{5}\right),\left(q, h_{5}\right),\left(q, h_{6}\right)$, $\left(b, h_{6}\right)$ and $\left(h_{1}, h_{5}\right)$ (blackened cells, Table 6$)$. It means that for some parameters sets the use of a third topography can be beneficial for the identification and have a slight adverse effect for other ones. 


\begin{tabular}{c|ccccccccc}
\hline$I_{z^{1}, z^{2}}$ & & & & & & & & & \\
$I_{\mathbf{z}^{1}, \mathbf{z}^{2}, \mathbf{z}^{3}}$ & $r_{0}$ & $q$ & $b$ & $h_{1}$ & $h_{2}$ & $h_{3}$ & $h_{4}$ & $h_{5}$ & $h_{6}$ \\
\hline$r_{0}$ & & 0.9 & 0.2 & 1.0 & 1.8 & 0.3 & 0.9 & 0.7 & 0.7 \\
$q$ & 1.0 & & 0.3 & 0.9 & 1.6 & 0.4 & 0.9 & 0.3 & 0.3 \\
$b$ & 0.2 & 0.3 & & 1.1 & 1.9 & 1.1 & 1.0 & 0.5 & 0.5 \\
$h_{1}$ & 0.7 & 0.6 & 0.6 & & 0.9 & 1.2 & 1.0 & 0.7 & 0.7 \\
$h_{2}$ & 0.3 & 0.2 & 0.4 & 0.5 & & 2.0 & 1.0 & 1.4 & 1.4 \\
$h_{3}$ & 0.3 & 0.3 & 1.2 & 0.6 & 0.4 & & 1.1 & 0.9 & 0.8 \\
$h_{4}$ & 0.9 & 0.7 & 0.9 & 0.5 & 0.6 & 1.0 & & 0.7 & 0.7 \\
$h_{5}$ & 0.8 & 0.6 & 0.5 & 0.9 & 0.4 & 0.7 & 0.7 & & 1.1 \\
$h_{6}$ & 0.7 & 0.4 & 0.6 & 0.2 & 0.3 & 0.8 & 0.6 & 0.6 & \\
\hline
\end{tabular}

Table 6 - $I$-index for all the parameter pairs using two and three imprint topographies $\mathbf{Z}^{1}, \mathbf{Z}^{2}$ and $\mathbf{Z}^{3}: I \leq 2$ (green, potentially identifiable), $2<I \leq 3$ (orange, difficult to identify), $I>3$ (red, not identifiable). Blackened cells highlight the $I$-index slightly increasing using a third topography.

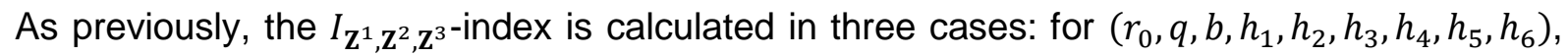
$\left(r_{0}, q, b\right)$ and $\left(h_{1}, h_{2}, h_{3}, h_{4}, h_{5}, h_{6}\right)$ (Figure 17). In all three cases, the use of a third imprint topography $\mathbf{Z}^{3}$ significantly reduces the index values.

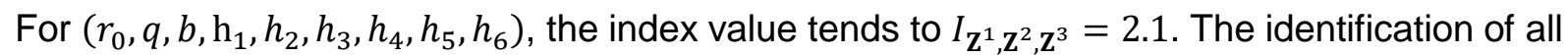
plastic parameters using the three imprint topographies is almost possible in the case where the material behaviour to identify is very close to the virtual material.

In the case where the interaction matrix components $\left(h_{1}, h_{2}, h_{3}, h_{4}, h_{5}, h_{6}\right)$ are known, $I_{\mathbf{Z}^{1}, \mathbf{Z}^{2}, \mathbf{Z}^{3}}\left(r_{0}, q, b\right)=1.0$, which ensures the identification of the isotropic hardening parameters if the material behaviour is very close to the virtual material and if all the other parameters are known. However, the index tends to a smaller value if two topographies are used $\left(\mathrm{I}_{\mathbf{Z}^{1}, \mathbf{Z}^{2}}\left(r_{0}, q, b\right)=0.9\right)$. One can notice that $I_{\mathbf{Z}^{1}, \mathbf{Z}^{2}, \mathbf{Z}^{3}}\left(r_{0}, q, b\right)$ value increases if the pile-ups of the third topography are included in the calculation. Once again, it is proved that the use of a third topography can have an adverse effect on the parameters identification, even if the effect is low in that case.

In the opposite case, where the isotropic hardening parameters $\left(r_{0}, q, b\right)$ are known, $\mathrm{I}_{\mathbf{Z}^{1}, \mathbf{Z}^{2}, \mathbf{Z}^{3}}\left(h_{1}, h_{2}, h_{3}, h_{4}, h_{5}, h_{6}\right)=1.7$. The identification of the interaction matrix can now be envisaged using the three topographies in the case where the material behaviour to identify is close to the virtual material and the hardening parameters are known. 
The pile-ups affect the index evolution. The slope of the index evolution is affected around $k=$ $14.10^{4}$ due to pile-ups of the third topography $\mathbf{Z}^{3}$ (Figure 17 ). Pile-ups mostly improve the conditioning of the inverse problem, but slightly degrade it sometimes.

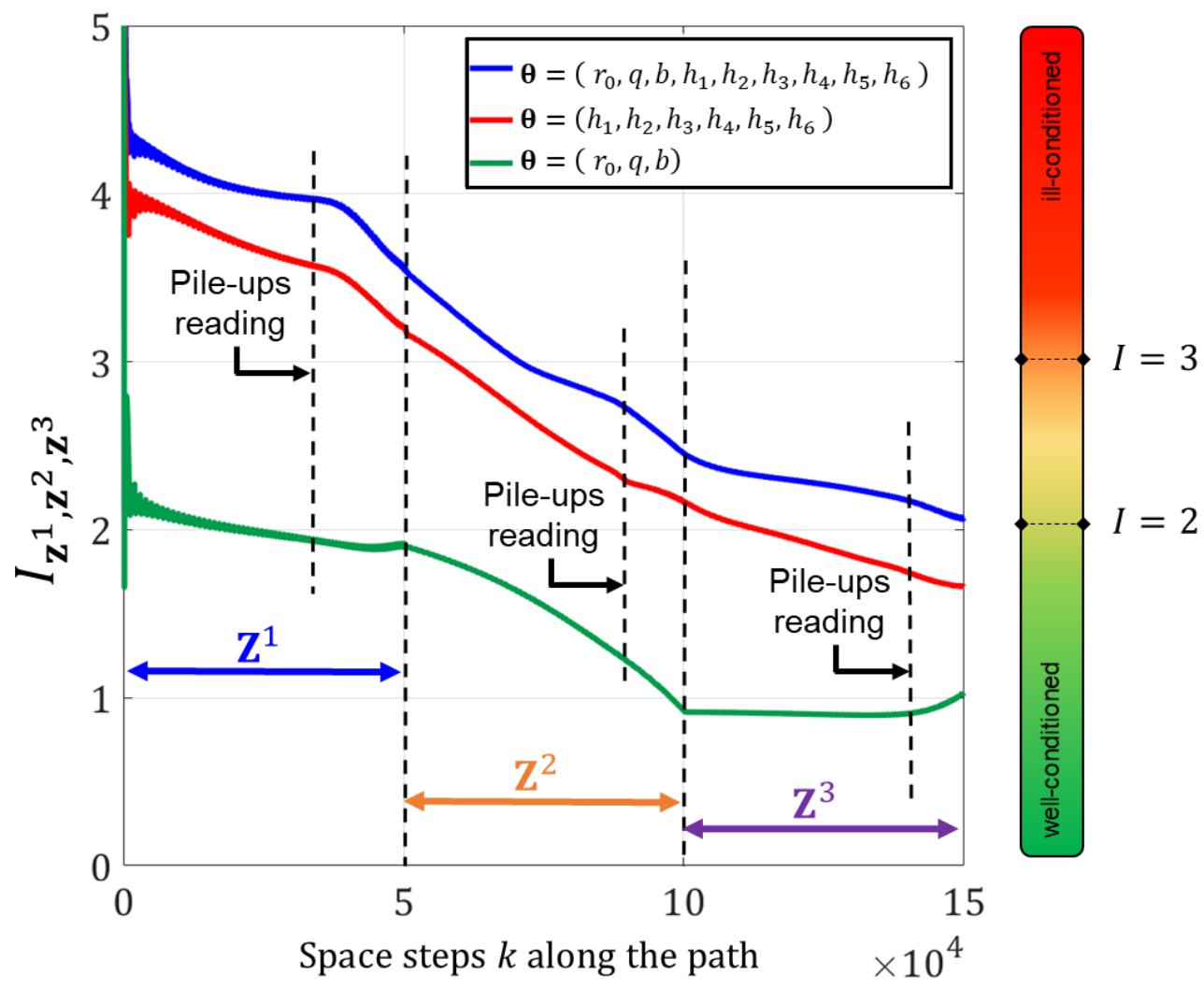

Figure $17-I_{\mathbf{Z}^{1}, \mathbf{Z}^{2}, \mathbf{Z}^{3}}$ evolution over the three topographies $\mathbf{Z}^{1}, \mathbf{Z}^{2}$ and $\mathbf{Z}^{3}$ for: all parameters (blue), isotropic hardening parameters $\left(r_{0}, q, b\right)$ only (green) and the interaction matrix components $\left(h_{1}, h_{2}, h_{3}, h_{4}, h_{5}, h_{6}\right)$ only (red). 


\section{Conclusions}

The identifiability of the parameters of the Méric-Cailletaud single crystal plasticity law using indentation curves and residual topographies has been evaluated. This method attempts to define the best-posed inverse problem which would ensure the parameters identification using the CPFEM updating method. In order to obtain a stable solution from the CPFEM updating procedure, an identifiability index is used for quantifying the information richness contained in the simulated responses, which means if the inverse problem is well-posed or ill-posed. The index reports the possible multicollinearity between the observation sensitivity vectors to parameters and the too large gap between the norms of sensitivity vectors to parameters, which can lead to an unstable FEM updating solution.

The identifiability of the Méric-Cailletaud single crystal plasticity law has been studied around the virtual material. The interaction matrix is fully populated and promotes the $h_{3}$ latent interaction component (Hirth locks). Firstly, the sensitivity vectors to the parameters have been calculated using the simulated loading indentation curve $\mathbf{P}$ only, performed in the [101] grain orientation, with an azimuth $\alpha_{[101]}^{[010]}=74^{\circ}$. The same process has been performed using the simulated imprint topography $\mathbf{Z}^{1}$ only, in the same conditions. Then, two simulated topographies $\mathbf{Z}^{1}$ and $\mathbf{Z}^{2}$ (performed in the [001] grain orientation with $\alpha_{[001]}^{[100]}=77^{\circ}$ ) have been used. Finally, a third imprint topography $\mathbf{Z}^{3}$ has been added to the process (performed in the [111] grain orientation with $\alpha_{[111]}^{[\overline{1} 2 \overline{1}]}=65^{\circ}$ ). The identifiability index is calculated in all the four cases and the Table 7 summarizes the minimum and maximum values for all the possible parameters combinations of the Méric-Cailletaud single crystal plasticity law.

It is well-known that the indentation curve is really poor in information for the parametric identification of material behaviour law at macroscopic scale (Cheng and Cheng, 1998). The present results show that if the parameters number exceeds 3 the identification of the MéricCailletaud law plastic parameters is impossible. For some parameters pairs, the identification is even difficult ( $I=2.9$ ) (Table $7,1^{\text {st }}$ column).

Identifiability results using only one imprint topography are better compared to those obtained with the use of the sole indentation curve. However, it has been shown that the identification of more than 6 parameters is not possible and at best difficult for 4 to 6 parameters (Table 7 , $2^{\text {nd }}$ column $)$. Except for $\left(b, h_{2}\right),\left(b, h_{3}\right),\left(h_{1}, h_{4}\right),\left(h_{1}, h_{6}\right)$ and $\left(h_{2}, h_{3}\right)$, all parameters pairs are identifiable using the imprint topography $\mathbf{Z}^{1}$.

The use of a second topography $\mathbf{Z}^{2}$ ensures all the parameter pairs identifiability of the MéricCailletaud law. In the worst case, the identifiability is difficult for more than 2 parameters. Some sets of 3, 4, 5, 6 and 7 parameters are identifiable (Table 7, $3^{\text {rd }}$ column). The index is always higher than 2 since $h_{2}$ and $h_{3}$ are part of the parameter vector studied (not shown here). In 
fact, the pair $\left(h_{2}, h_{3}\right)$ gives the worst identifiability index $\mathrm{I}_{\mathbf{Z}^{1}, \mathbf{Z}^{2}}\left(h_{2}, h_{3}\right)=2.0$. According to the results in dislocation dynamics (Madec, 2001), $h_{2}$ refers to the interaction with a low hardening (coplanar interaction). One can assume that these interactions have a little effect on the imprint topography and are difficult to measure. In fact, the sensitivity of the chosen simulated topographies to $h_{2}$ has always the lowest norm. In contrast, according to the sensitivity analysis, $h_{3}$ strongly affects topographies but the sensitivity vector to $h_{3}$ is certainly collinear with respect to the sensitivity vector to $h_{2}$. Moreover, the norm of the sensitivity vectors associated to $h_{2}$ and $h_{3}$ do not have the same magnitude at all. The ratio between both norms is around 12.6, which predicts that the identifiability of $\left(h_{2}, h_{3}\right)$ could be very difficult. It is also interesting to notice that the inverse problem using both topographies $\mathbf{Z}^{1}$ and $\mathbf{Z}^{2}$ to identify 9 parameters is as well-posed as that using topography $\mathbf{Z}^{1}$ only to identify the pair $\left(b, h_{3}\right)$ $(\mathrm{I}=2.4)$.

Finally, a third topography $\mathbf{Z}^{3}$ is added to the identifiability analysis. It ensures the identifiability of all parameters combinations up to 8 parameters. For a full parameter set ( 9 parameters), $I_{\mathbf{z}^{1}, \mathbf{z}^{2}, \mathbf{z}^{3}}=2$.1. Thus, the inverse problem using the three topographies to identify 9 parameters $\left(I_{\mathbf{z}^{1}, \mathbf{z}^{2}, \mathbf{z}^{3}}=2.1\right)$ is almost as well-posed as that using two topographies $\mathbf{Z}^{1}$ and $\mathbf{Z}^{2}$ to identify the pair $\left(h_{2}, h_{3}\right)\left(\mathrm{I}_{\mathbf{Z}^{1}, \mathbf{Z}^{2}}=2.0\right)$.

Generally, it can be concluded that the topographies, and especially pile-ups, tend to improve the conditioning of the inverse problem. In many cases the non-uniqueness is an extreme case of sensitivity to experimental errors (i.e. ill-conditioned inverse problem) (Phadikar et al., 2013). The $I$-index approach is interesting to avoid this difficulty and design the cost function used in the updating method. The $I$-index can be used for choosing the best indenter/grain relative orientation to indent. Thus, it could be possible to identify all or part of the parameters of MéricCailletaud single crystal plasticity law. But for this, the material behaviour to identify must be close to the virtual material used. The final verification of uniqueness using several starting points of a local minimization algorithm remains anyway necessary. 


\begin{tabular}{c|cccc}
\hline $\begin{array}{c}p \text {-combination } \\
\text { of plastic parameters }\end{array}$ & $\begin{array}{c}I_{\mathbf{p}} \\
{[\min , \max ]}\end{array}$ & $\begin{array}{c}I_{\mathbf{z}^{1}} \\
{[\min , \max ]}\end{array}$ & $\begin{array}{c}I_{\mathbf{z}^{1}, \mathbf{z}^{2}} \\
{[\min , \max ]}\end{array}$ & $\begin{array}{c}I_{\mathbf{z}^{1}, \mathbf{z}^{2}, \mathbf{z}^{3}} \\
{[\mathrm{~min}, \max ]}\end{array}$ \\
\hline 2 & {$[0.1 ; 2.9]$} & {$[0.3 ; 2.4]$} & {$[0.2 ; 2.0]$} & {$[0.2 ; 1.2]$} \\
3 & {$[1.1 ; 5.5]$} & {$[1.3 ; 3.1]$} & {$[0.5 ; 2.3]$} & {$[0.4 ; 1.6]$} \\
4 & $>6$ & {$[2.1 ; 3.4]$} & {$[1.2 ; 2.3]$} & {$[0.7 ; 1.8]$} \\
5 & $>6$ & {$[2.3 ; 3.5]$} & {$[1.4 ; 2.4]$} & {$[1.0 ; 1.9]$} \\
6 & $>6$ & {$[2.7 ; 3.5]$} & {$[1.8 ; 2.4]$} & {$[1.2 ; 1.9]$} \\
7 & $>6$ & {$[3.1 ; 3.5]$} & {$[1.9 ; 2.4]$} & {$[1.5 ; 2.0]$} \\
8 & $>6$ & {$[3.3 ; 3.5]$} & {$[2.1 ; 2.4]$} & {$[1.9 ; 2.0]$} \\
9 & $>6$ & 3.6 & 2.4 & 2.1 \\
\hline
\end{tabular}

Table 7 - $I$-index values for all parameters combinations using: the indentation curve $\mathbf{P}$ only $\left(I_{\mathbf{P}}\right)$, the topography $\mathbf{Z}^{1}$ only $\left(I_{\mathbf{Z}^{1}}\right)$, combination of two topographies $\left(\mathbf{Z}^{1}, \mathbf{Z}^{2}\right)$ and the three topographies $\left(\mathbf{Z}^{1}, \mathbf{Z}^{2}, \mathbf{Z}^{3}\right): I \leq 2$ (green, potentially identifiable), $2<I \leq 3$ (difficult to identify), $I>3$ (red, not identifiable).

A fourth imprint topography $\mathbf{Z}^{4}$ could maybe bring enough information to identify a full parameter set and provide a better index value $\left(I_{\mathbf{z}^{1}, \mathbf{z}^{2}, \mathbf{z}^{3}, \mathbf{z}^{4}}\left(r_{0}, b, q, h_{1}, h_{2}, h_{3}, h_{4}, h_{5}, h_{6}\right) \leq 2.0\right)$. The indenter/grain relative orientations space could be screened to look for the best complementary topographies. As Zambaldi and Raabe did (Zambaldi and Raabe, 2010), a map of the richest orientations for the parametric identifiability study could be built.

As Kysar et al. (Kysar et al., 2007) or Breumier et al. (Breumier et al., 2019), it would be also interesting to measure the crystal lattice curvature induced at the free surface by the indentation by electron backscatter diffraction (EBSD). The information richness of crystal lattice curvature around imprints obtained after Berkovich nanoindentation could be measured using the process reported in the present paper.

The parametric identifiability has been studied around the chosen virtual material which promotes the $h_{3}$ latent interaction component (Hirth locks). The analysis must be extended to other virtual materials (Renner et al., 2016). According to results in dislocation dynamics, $h_{4}$ refers to the interaction with the highest hardening (collinear interaction) (Fivel, 1997; Fivel et al., 1997; Madec, 2001; Madec et al., 2003; Devincre et al., 2006). It would be interesting to perform the same process around a virtual material which promotes the collinear interactions and to extend the analysis to the nickel cold-worked behaviour of (Renner et al., 2016) in order to analyse the effect of the hardening on the identifiability index. 


\section{Acknowledgement}

This research was supported by the French Ministry of Higher Education and Research. 


\section{References}

Alkorta, J., Martínez-Esnaola, J.M., Sevillano, J.G., 2005. Absence of one-to-one correspondence between elastoplastic properties and sharp-indentation loadpenetration data. J. Mater. Res. 20, 432-437.

Bocciarelli, M., Bolzon, G., Maier, G., 2005. Parameter identification in anisotropic elastoplasticity by indentation and imprint mapping. Mech. Mater. 37, 855-868.

Bolzon, G., Buljak, V., Maier, G., Miller, B., 2011. Assessment of elastic-plastic material parameters comparatively by three procedures based on indentation test and inverse analysis. Inverse Probl. Sci. Eng. 19, 815-837.

Bolzon, G., Maier, G., Panico, M., 2004. Material model calibration by indentation, imprint mapping and inverse analysis. Int. J. Solids Struct. 41, 2957-2975.

Breumier, S., Villani, A., Maurice, C., Lévesque, M., Kermouche, G., 2019. Effect of crystal orientation on indentation-induced residual stress field: Simulation and experimental validation. Mater. Des. 169, 107659.

Burlet, H., Cailletaud, G., 1991. Zebulon a finite element code for non-linear material behaviour, in: European Conf. on New Advances in Computational Structure Mechanics, Giens, France.

Cao, Y., 2004. Depth-sensing instrumented indentation with dual sharp indenters: stability analysis and corresponding regularization schemes. Acta Mater. 52, 1143-1153.

Capehart, T.W., Cheng, Y.T., 2003. Determining constitutive models from conical indentation: Sensitivity analysis. J. Mater. Res. 18, 827-832.

Casals, O., Alcalá, J., 2005. The duality in mechanical property extractions from Vickers and Berkovich instrumented indentation experiments. Acta Mater. 53, 3545-3561.

Charleux, L., 2006. Micromécanique de l'essai d'indentation: expériences et simulations. Grenoble, INPG.

Chen, X., Ogasawara, N., Zhao, M., Chiba, N., 2007. On the uniqueness of measuring elastoplastic properties from indentation: The indistinguishable mystical materials. J. Mech. Phys. Solids 55, 1618-1660.

Cheng, Y.-T., Cheng, C.-M., 1999. Can stress-strain relationships be obtained from indentation curves using conical and pyramidal indenters? J. Mater. Res. 14, 34933496. https://doi.org/10.1557/JMR.1999.0472

Cheng, Y.-T., Cheng, C.-M., 1998. Analysis of indentation loading curves obtained using conical indenters. Philos. Mag. Lett. 77, 39-47.

Devincre, B., Kubin, L., Hoc, T., 2006. Physical analyses of crystal plasticity by DD simulations. Scr. Mater. 54, 741-746. 
Fivel, M., 1997. Etudes numériques à différentes échelles de la déformation plastique des monocristaux de structure CFC.

Fivel, M., Tabourot, L., Rauch, E., Canova, G., 1998. Identification through mesoscopic simulations of macroscopic parameters of physically based constitutive equations for the plastic behaviour of fcc single crystats. J. Phys. IV 08, Pr8-151-Pr8-158.

Fivel, M., Verdier, M., Canova, G., 1997. 3D simulation of a nanoindentation test at a mesoscopic scale. Mater. Sci. Eng. A 234-236, 923-926.

Forest, S., 2010. Continuum crystal plasticity theory, in: Mechanics of Nano-Objects. Presses Mines ParisTech, pp. 101-116.

Forest, S., Fivel, M., 2001. Modeles discrets et continus de la plasticité des métaux: du monocristal au polycristal. Ecole Thématique St Pierre D’Oléron 457-466.

Francavilla, A., Zienkiewicz, O.C., 1975. A note on numerical computation of elastic contact problems. Int. J. Numer. Methods Eng. 9, 913-924.

Franciosi, P., 1985. The concepts of latent hardening and strain hardening in metallic single crystals. Acta Metall. 33, 1601-1612.

Gérard, C., 2008. Mesures de champs et identification de modèles de plasticité cristalline. Université Paris-Nord-Paris XIII.

Gérard, C., Bacroix, B., Bornert, M., Cailletaud, G., Crépin, J., Leclercq, S., 2009. Hardening description for FCC materials under complex loading paths. Comput. Mater. Sci. 45, 751-755.

Gérard, C., Cailletaud, G., Bacroix, B., 2013. Modeling of latent hardening produced by complex loading paths in FCC alloys. Int. J. Plast. 42, 194-212.

Guery, A., 2014. Développement d'une méthode de corrélation d'images numériques adaptée aux mesures cinématiques dans les polycristaux: application à l'identification de paramètres de lois de plasticité cristalline. ENS Cachan.

Guery, A., Latourte, F., Hild, F., Roux, S., 2014. Identification of crystal plasticity law parameters using kinematic measurements in polycrystals, in: WCCM XI-11th World Congress on Computational Mechanics. pp. 8-pp.

Guilhem, Y., 2011. Étude numérique des champs mécaniques locaux dans les agrégats polycristallins d'acier $316 \mathrm{~L}$ sous chargement de fatigue. École Nationale Supérieure des Mines de Paris.

Gujarati, D.N, 1988. Basic econometrics, 2 nd. ed. McGraw-Hill, New York.

Harding, J.W., Sneddon, I.N., 1945. The elastic stresses produced by the indentation of the plane surface of a semi-infinite elastic solid by a rigid punch, in: Mathematical Proceedings of the Cambridge Philosophical Society. Cambridge Univ Press, pp. 1626. 
Jean, M., 1995. Frictional contact in collections of rigid or deformable bodies: numerical simulation of geomaterial motions. Mech. Geomaterial Interfaces 463.

Kysar, J.W., Gan, Y.X., Morse, T.L., Chen, X., Jones, M.E., 2007. High strain gradient plasticity associated with wedge indentation into face-centered cubic single crystals: geometrically necessary dislocation densities. J. Mech. Phys. Solids 55, 1554-1573.

Ladeveze, P., 1980. Sur la théorie de la plasticité en grandes déformations. ENS-Cachan-LMT Intern. Rep.

Lee, E.H., 1969. Elastic-plastic deformation at finite strains. J. Appl. Mech. 36, 1-6.

Ma, Z.S., Zhou, Y.C., Long, S.G., Zhong, X.L., Lu, C., 2012. Characterization of stress-strain relationships of elastoplastic materials: An improved method with conical and pyramidal indenters. Mech. Mater. 54, 113-123.

Madec, R., 2001. Des intersections entre dislocations à la plasticité du monocristal CFC: étude par dynamique des dislocations. Paris 11, Orsay.

Madec, R., Devincre, B., Kubin, L., Hoc, T., Rodney, D., 2003. The role of collinear interaction in dislocation-induced hardening. Science 301, 1879-1882.

Méric, L., Cailletaud, G., Gaspérini, M., 1994. FE calculations of copper bicrystal specimens submitted to tension-compression tests. Acta Metall. Mater. 42, 921-935.

Oliver, W.C., Pharr, G.M., 1992. An improved technique for determining hardness and elastic modulus using load and displacement sensing indentation experiments. J. Mater. Res. 7, 1564-1583.

Pac, M.-J., Giljean, S., Rousselot, C., Richard, F., Delobelle, P., 2014. Microstructural and elasto-plastic material parameters identification by inverse finite elements method of $\mathrm{Ti}$ $(1-x)$ Al x N (0. Thin Solid Films 569, 81-92.

Phadikar, J.K., Bogetti, T.A., Karlsson, A.M., 2013. On the uniqueness and sensitivity of indentation testing of isotropic materials. Int. J. Solids Struct. 50, 3242-3253.

Pottier, T., 2010. Modélisation multiéchelle du comportement et de l'endommagement des composites à matrice métallique. Marne-la-Vallée, ENPC.

Rao, J.G., Varma, S.K., 1993. The Effect of Grain Size and Strain Rate on the Substructures and Mechanical Properties in Nickel 200. Metall. Trans. A 24, 2559-2568.

Renner, E., 2016. Vers l'identification d'une loi de plasticité monocristalline par analyse topographique d'empreintes de nanoindentation Berkovich ( $\mathrm{PhD}$ Thesis). Université de Franche Comté.

Renner, E., Gaillard, Y., Richard, F., Amiot, F., Delobelle, P., 2016. Sensitivity of the residual topography to single crystal plasticity parameters in Berkovich nanoindentation on FCC nickel. Int. J. Plast. 77, 118-140.

Richard, F., Villars, M., Thibaud, S., 2013. Viscoelastic modeling and quantitative experimental characterization of normal and osteoarthritic human articular cartilage using 
indentation. J. Mech. Behav. Biomed. Mater. 24, 41-52. https://doi.org/10.1016/j.jmbbm.2013.04.012

Sachdeva, T.D., Ramakrishnan, C.V., 1981. A finite element solution for the two-dimensional elastic contact problems with friction. Int. J. Numer. Methods Eng. 17, 1257-1271.

Schwartz, J., 2011. Approche non locale en plasticité cristalline: application à l'étude du comportement mécanique de l'acier AISI 316LN en fatigue oligocyclique. ChâtenayMalabry, Ecole centrale de Paris.

Sneddon, I.N., 1965. The relation between load and penetration in the axisymmetric Boussinesq problem for a punch of arbitrary profile. Int. J. Eng. Sci. 3, 47-57.

Taljat, B., Zacharia, T., Kosel, F., 1998. New analytical procedure to determine stress-strain curve from spherical indentation data. Int. J. Solids Struct. 35, 4411-4426.

Tasan, C.C., Diehl, M., Yan, D., Zambaldi, C., Shanthraj, P., Roters, F., Raabe, D., 2014a. Integrated experimental-simulation analysis of stress and strain partitioning in multiphase alloys. Acta Mater. 81, 386-400.

Tasan, C.C., Hoefnagels, J.P.M., Diehl, M., Yan, D., Roters, F., Raabe, D., 2014b. Strain localization and damage in dual phase steels investigated by coupled in-situ deformation experiments and crystal plasticity simulations. Int. J. Plast. 63, 198-210.

Tho, K.K., Swaddiwudhipong, S., Liu, Z.S., Zeng, K., Hua, J., 2004. Uniqueness of reverse analysis from conical indentation tests. J. Mater. Res. 19, 2498-2502.

Vlassak, J.J., Nix, W.D., 1994. Measuring the elastic properties of anisotropic materials by means of indentation experiments. J. Mech. Phys. Solids 42, 1223-1245.

Vlassak, J.J., Nix, W.D., 1993. Indentation modulus of elastically anisotropic half spaces. Philos. Mag. A 67, 1045-1056.

Wronski, M., 1994. Couplage du contact et du frottement avec la mécanique non linéaire des solides en grandes déformations. Application à l'étude des blocs de mousse en polyuréthane.

Zambaldi, C., Raabe, D., 2010. Plastic anisotropy of $\mathrm{y}$-TiAl revealed by axisymmetric indentation. Acta Mater. 58, 3516-3530.

Zambaldi, C., Yang, Y., Bieler, T.R., Raabe, D., 2012. Orientation informed nanoindentation of a-titanium: Indentation pileup in hexagonal metals deforming by prismatic slip. J. Mater. Res. 27, 356-367.

Zambaldi, C., Zehnder, C., Raabe, D., 2015. Orientation dependent deformation by slip and twinning in magnesium during single crystal indentation. Acta Mater. 91, 267-288.

Zhao, M., Chen, X., Xiang, Y., Vlassak, J.J., Lee, D., Ogasawara, N., Chiba, N., Gan, Y.X., 2007. Measuring elastoplastic properties of thin films on an elastic substrate using sharp indentation. Acta Mater. 55, 6260-6274.

Z-set User commands Version 9.0, 2018. . ENSMP-Northwest Numerics-ONERA. 